\title{
Phenomenological Strands for Gaming Disorder and Esports Play: A Qualitative Registered Report
}

\author{
Stage 2, (version 2)
}

Veli-Matti Karhulahti ${ }^{1 *}$, Miia Siutila ${ }^{1,2}$, Jukka Vahlo, ${ }^{1}$ Raine Koskimaa ${ }^{1}$

\footnotetext{
${ }^{1}$ University of Jyväskylä, Jyväskylä, Finland

${ }^{2}$ University of Turku, Turku, Finland

*Corresponding author: Veli-Matti Karhulahti (vmmkar@utu.fi)
}

Accepted Stage 1: https://rr.peercommunityin.org/articles/rec?id=44

Preregistered Stage 1 protocol: https://osf.io/a2rwg (date of in-principle acceptance: 24/09/2021)

Note: Stage 1 preregistration includes a 1-year follow-up study with the same participants. The study is underway and will be completed in early 2023 by estimate.

\section{Abstract}

The recent inclusion of gaming disorder in the ICD-11 as a mental disorder has further increased the importance of researching the health spectrum related to gaming. A critical area in this regard is the lack of clarity concerning the differences between gaming disorder and intensive play, the latter of which often involves several gaming hours per day without related health problems. In this study, we approached the above question by interpretive phenomenological analysis with interviews in two groups of highly involved videogame players: those who seek or have sought clinical help for their problems with gaming $(n=6)$, and those who play esports more than $\mathbf{4}$ hours per day without self-reported related health problems $(n=10)$. The interviews were carried out by using a new Phenomenology of Play (POP) interview frame. These data were contextualized with interviews of medical experts $(n=6)$ who have experience of working with the former group. The findings imply that experiences of disorder derive from gaming interfering with what one wants to be, do, and have throughout life, whereas the experiences of intensive esport play derive from gaming being integrated into self throughout life. To our knowledge, this was the first registered report using qualitative phenomenological methods-we encourage researchers to explore the utility of registered reports with other qualitative methods to further optimize scientific progress. 


\section{Introduction}

For a long time, scholars have known play to be a significant component of human life. From childhood development (Piaget 2013) to adolescence growth (Pellegrini 2009) and adult recreation (van Leeuwen \& Westwood 2008), various forms of playing belong to our lifecycles. Today, research on the topic is complicated by videogame play-gaming-which has become a popular type of play globally across age groups.

Many studies have found evidence indicating gaming to contribute to life quality. For instance, a large nationally representative study of children between 10 and 15 years found those who play videogames daily have better psychosocial adjustment compared with the non-gaming group (Przybylski 2014). Similar results have also been found with adolescents and adults who can find videogames supportive for social health (Kowert, Domahidi \& Quandt 2015), wellbeing more generally (Johannes, Vuorre \& Przybylski 2021), and even gaming mediated psychotherapy (Ceranoglu 2010). Gaming is widely considered a potential means to several cognitive, emotional, and motivational benefits (Granic, Lobel \& Engels 2014).

At the same time, negative associations between gaming and health have become an emergent research topic. In 2013, the American Psychiatric Association (APA) cautiously announced 'internet gaming disorder' in their Diagnostic and Statistical Manual of Mental Disorders ( $5^{\text {th }}$ edition) as a condition in need for further study. In 2019, the World Health Organization (WHO) took a more drastic step and confirmed 'gaming disorder' to be included in their International Classification of Diseases (11 ${ }^{\text {th }}$ edition) as a mental disorder due to addictive behaviors. Consequently, an increasingly large body of varied research has emerged regarding the colloquially termed 'videogame addiction' and its epidemiology in particular (Kardefelt-Winther 2017; King et al. 2020; Van Rooij et al. 2017). Meanwhile, the nosology of this now-officialized mental disorder remains under active debate (Bean et al. 2017), and its ontology-what disorder-based gaming really is-largely unknown.

Considering the situation where gaming simultaneously gathers evidence for both negative and positive health associations, large groups of experts around the world systematically express confusion about the foundations of the two (Ferguson \& Colwell 2020). As for gaming disorder, a foremost yet still unsolved issue has been the inability to distinguish empirically and theoretically between highly involved healthy videogame players and those who suffer from genuine gaming-related health issues (Billieux et al. 2019; Charlton \& Danforth 2007; Nielsen \& Karhulahti 2017). The relationship of these health issues and gaming is further complicated by the lack of causal evidence and theory, as it remains unclear whether the associated functional impairment and stress derive from gaming or the other way around (e.g., Jeong et al. 2019; van Rooij et al. 2017); or alternatively, if these links could be explained by a network theoretical or some other approach (see Fried et al. 2017; Stavropoulos et al. 2019). In practice, the lack of good answers to these questions means both false positive and false negative diagnoses, which can be seriously harmful when over and under medicating (or otherwise treating) people in opposing life situations-especially in child and adolescent groups, where medical decisions are often made by caregivers and parents.

To make progress in solving these issues, a frequent recommendation by numerous experts has been to study gaming phenomenologically with both treatment-seeking and highly involved healthy players (Castro-Calvo et al. 2021; Colder Carras \& Kardefelt-Winther 2018; Griffiths et al. 2016). Although the literature in the area is now beginning to emerge (Colder Carras et al. 2018; Karhulahti 2020), no studies have investigated the phenomenological nature of treatment-seeking players' lived experiences in comparison to 
intensively gaming individuals who do not report related health problems. In this study, we do exactly that, with the latter group selected from the domain of amateur competitive gaming-esports-which has recently become one of the major forms of videogame play across cultures and a central point of related health discussions (Brevers, King \& Billieux 2020).

The ambiguities related to treatment-seeking players must be noted separately. In previous literature, such groups have been addressed as 'addicted players', 'problem players', and 'players with gaming disorder', among other similar labels. Because the literature relies mainly on surveys, the labels typically derive from screening tools that claim to measure 'videogame addiction', 'gaming disorder', and other such constructs, which may or may not be consistent with the diagnostic criteria in the ICD-11 (King et al. 2020; Karhulahti et al. 2021). It is beyond the scope of this study to assess the clinical status of our participantslongitudinal studies with clinical expert interviews are needed to investigate the health scenarios of gaming treatment-seekers (see Ko et al. 2020; Starcevic, et al. 2020)-therefore, the link between our study and gaming disorder (in the ICD-11) is determined by treatmentseeking alone. Although this is arguably a much stronger link than those established by screening tools (see Satchell et al. 2021; van Rooij et al. 2018), we stress that our participants may not meet diagnostic gaming disorder criteria despite their treatment-seeking. Likewise, our other group 'players without self-reported health problems related to gaming' was recruited based on self-reported health alone.

The general aim of this study was to discover the differences and similarities between the gaming experiences of treatment-seekers (potential gaming disorder) and those who selfreport no related health problems. The findings form a phenomenological basis thathopefully with future replications consisting of different samples-help model the differences between gaming disorder and healthy intensive play. Ultimately, this can improve both the accuracy of related screening instruments as well as the diagnostic understanding of gaming disorder at large. Our work was thus guided by the following research question:
RQ1: How do the experiences and meanings of playing videogames-shaped by the individuals' diverse types of sociocultural contexts-differ between those with related health problems (potential gaming disorder defined by treatment- seeking) and those who play esports games several hours per day without self- reported health problems related to gaming?

The research question is nonconfirmatory in the sense that we disclosed two qualitative hypotheses for it. By qualitative hypotheses we mean that the goal was not to seek confirming and falsifying evidence, and we do not claim the data to (not) support the alternative hypotheses (or null). This is because qualitative research, at least in the presently applied interpretive phenomenological form, is not well suited for null hypothesis significance testing. That said, we do consider phenomenological research suitable for another kind of significance testing-significance as meaning-and because these forms of significance already have an existing basis in the literature, disclosing this basis openly in the form of qualitative hypotheses added to the transparency of the work. Accordingly, we considered it important to set hypotheses not to test hypotheses, but rather because we wanted to disclose our hypothetical biases. 
First, with reference to a recent phenomenological study (Colder Carras et al. 2018) that asked active gamers "What are signs of video game addiction, Internet gaming disorder, or disordered gaming?" and found the participants ranking the 'neglect of needs/responsibilities' and 'loss of control' as critical signs, we expected our participants with related health problems to share experiences of these types. Since earlier research has also frequently suggested social difficulties to be related to gaming problems (Kneer et al. 2014; Kowert et al. 2015; Snodgrass et al. 2019), we expected these participants' experiences to occur in a distinct psychosocial context.

\begin{abstract}
QH1: We expect participants with gaming-related health problems (as defined by treatment-seeking) to talk about their gaming experiences in the context of neglected needs or responsibilities, loss of control, and social difficulties. For them, meaning(s) in gaming compensate for these issues. We do not expect our esports gaming participants (self-reporting no health problems related to gaming) to express the above.
\end{abstract}

Second, based on recent phenomenological anthropology (Karhulahti 2020), interview studies (Himmelstein, Liu \& Shapiro 2021; Siutila \& Karhulahti 2021), and qualitative online discussion analyses (Kou \& Gui 2020) that have found highly involved competitive videogame players to consider gaming meaningful to them-often manifested by commitment to selfdevelopment and perceived social value in the game (see also García-Lanzo \& Chamarro 2018)-we expected these elements to surface in the experiences of esports-playing participants. To further clarify the manifestations of these meanings: by self-development we mean activities, attitudes, and behaviors that aim at improving or progressing oneself as a player or participant in the game, and by perceived social value we mean gaming constituting a meaningful part of one's social identity, feelings of belonging, or social need satisfaction in general.

Although the literature on gaming disorder is more limited in this regard, at least one interview study (Shi et al. 2019) has suggested similar meaningfulness to be found by those who meet the DSM5 criteria for 'internet gaming disorder'. Hence, we expected the above elements also to be present in treatment-seekers' experiences. Because gaming disorder is conceptually based on significant clinical impairment and distress due to gaming, including social difficulties, we also expected significant life conflicts for the treatment-seeking players (see also Götzenbrucker \& Köhl 2009; King \& Delfabbro 2009; Karlsen 2013; Domahidi \& Quandt 2015; Snodgrass, et al. 2018).

QH2: We expect the esports gaming participants (self-reporting no gamingrelated health problems) and the participants with related health problems (as defined by treatment-seeking) to find gaming meaningful to their lives, as expressed by pursued self-development in their games as well as the social value given to it-but only the latter to experience significant life conflicts.

To investigate all the above, we conducted a comparative interview study by means of interpretive phenomenological analysis (IPA) with two primary participant groups: those recruited from local hospitals, therapy centers, and other medical institutions with gamingrelated health problems (as defined by treatment-seeking) and those recruited from local gaming communities with intensive esports play habits but no related health problems (as 
defined by self-report). As an idiographic approach that allows for detailed investigation of complex experiences and even existential depth (Smith et al. 2009), IPA was an efficient means to seek answers to the above. In addition, with the help of our collaborating institutions, we recruited medical experts for semi-structured interviews to exploratively contextualize and assess the differences between the treatment-seeking participants' personal experiences and the views of those who treat them. Following the ongoing development of qualitative preregistration protocols and open science practices (Haven et al. 2020; Haven \& Van Grootel 2019), the interview transcripts were submitted to the Finnish Social Science Data Archive (FSD) for open scientific use.

\section{Methods}

\section{Ethics information}

The study was carried out according to a project plan funded by the Finnish Work Environment Fund. The research and data management plans received a positive appraisal from the Human Sciences and Ethics Committee in the University of Jyväskylä. The research was carried out according to the Finnish National Board on Research Integrity guidelines and the Helsinki Declaration with its later amendments. Informed consent was collected from all participants. By signing the consent form, the participants agreed to share their interview transcripts for later scientific use via the FSD. It was highlighted for the participants that the risks involved in this data sharing are of different mode compared to, for instance, surveybased quantitative data sharing (Karhulahti 2021).

Personal identifiers were removed from the transcripts carefully in a 4-step process: initial transcription, transcript editing, participant checking, and finally the processing of the transcripts for sharing by the FSD [ongoing]. When participants wished not to share some transcript sections, these sections were kept unshared. Considering the challenges of finding consenting participants (Branney et al. 2019), we decided to share the data via the FSD, an experienced and one of the leading repositories for sharing qualitative data (Bishop \& KuulaLuumi 2017), which helped in recruitment. Allowing the participants to review their transcripts built further confidence and trust. The data are stored pseudonymously until the end of the project following the GDPR; the need of the pseudonym key will be reevaluated regularly and when it is no longer needed, it will be destroyed, making the data anonymous. The participants also had an option to share their transcripts without anonymization. Being able to share reusable qualitative data is especially helpful with hard-to-reach respondents, as collecting such data is usually laborious for both researchers and participants. The below methodological details are further supplemented by a 32-point list of consolidated criteria for reporting qualitative research (Appendix 1).

\section{Participants}

Two types of intensively gaming participants form the core groups. Additionally, a group of medical experts were interviewed. A more detailed explanation of the groups and their role in the study is below. All participants were recruited simultaneously. The inclusion and exclusion criteria were mentioned in the call, and the participants' fit was double checked before the interview in private communication. The analysis of the transcripts was conducted 
by stages (see analysis plan). All participants had to live or work in Finland; one participant was interviewed in English and others in Finnish. Because our work concerns the adult working population, full-time students were excluded from all groups.

Sample justification. The participants' experiences were investigated in a semistructured interview with a Phenomenology of Play frame (see below) via interpretive phenomenological analysis (IPA). Unlike in many other forms of qualitative inquiry, data saturation "does not make sense to phenomenology" (Van Manen, Higgins \& Van der Reit 2016) because there is no saturation point for phenomenological meaning. This premise was recently supported by a systematic assessment of interview data saturation (Hennink, Kaiser \& Marconi 2017). In IPA research where the goal is to understand meanings in particularly complex experiences, it is unlikely that any number of interviews would ever saturate the data in terms of theoretical or other common forms of saturation (Saunders et al. 2018, see also Braun \& Clarke 2020). Therefore, the rationale for our sample was based on a) the specific nature of IPA research, b) the limitations concerning the object of study, and c) the practical confines set by our funding and ethical committee approval.

a) In IPA, which we apply, samples are recommended to be small and homogenous because the primary idea is to study how a specific type of experience (e.g., gaming disorder) is personally experienced (Smith 1996; Smith, Jarman \& Osborn 1999). Whereas general IPA sample size recommendations differ between $N=1$ and $N=15$ per topic, an average number of study participants has been estimated as six (Smith \& Osborn 2007). Following the above, it is not helpful to think in terms of random or representative sampling; rather, the topic defines the boundaries of the relevant sample and its characteristics (Larkin \& Thompson 2012).

b) Our study concerns gaming disorder, which in diagnostic practice is a new phenomenon. Cultural differences are of utmost significance here, nevertheless. For instance, in South Korea the government organizes systematic survey screening of gaming problems for local students annually, and those in the screening-based risk group are guided to receive an intervention (Jeong et al. 2018). This means that countries like South Korea can have thousands of individuals in medical interventions due to screening-based disorder risk. That said, due to the screening tools' tendency to over-pathologize healthy individuals (Boonen et al. 2018; Ferguson, Coulson \& Barnett 2011) and considering South Korea's specific cultural context where it is common for parents to interfere with their children's gaming (Carbonell 2017; Jeong, Ferguson \& Lee 2019), a large part of the individuals participating in such intervention do not suffer from actual clinically significant symptoms (Starcevic et al. 2020). In Finland, in turn, medical services for gaming-related health problems are scarce and rarely used (see below), and the Finnish government does not screen schools or other institutions for gaming problems. This implies (but does not confirm) that people who end up seeking related medical support in Finland suffer from actual, clinically significant symptoms. In this project, we collaborate with two such major medical service providers, and their combined overall number of annual treatment-seekers roughly coheres with the recommended sample size of an IPA study (personal communication with our collaborators during project planning). Thus, even though we are not limited to the treatment-seekers of our two collaborators, there are good reasons to believe that the small number of actual gaming-related 
treatment-seekers in Finland will be a pragmatic element that sets sample size boundaries for this study as well. Finding a larger-than-recommended (more than $n=15$ ) sample of players with serious gaming-related health problems would require more time and resources. On the other hand, the probability that our participants suffer from genuine distress and functional impairment related to gaming is high.

c) Last, and related to the above, we note that this research was funded by the Finnish Work Environment Fund for years 2021 and 2022. As usual, in the application phase, we were required to report a priori sample size estimations, and the resources of the project were allocated to carry out two rounds of phenomenological interviews $(2021,2022)$ based on the recommended IPA sample sizes $(N=5-15)$ for each year, respectively. Our ethical committee approval is based on this research plan. With more resources and time, it would be possible to find and recruit larger sample(s) that could further expand the data and possible findings; however, in the present study we are committed to carry out a study in a way that has been funded and ethically approved.

In summary, our sample size concerning people with gaming-related health problems-as defined by their treatment-seeking in the Finnish context where clinical treatment is scarcely provided and rarely sought-is based on (a) the current recommendations ( $N=1-15)$ of IPA methodology, (b) the topic in question with the specific sociocultural context taken into consideration, and $(c)$ the practical boundaries of time, resources, and a priori project plan that has been approved by the ethics committee. Below, we describe each of the three interview groups in detail to further elaborate on their recruitment and role in the study.

Group 1. This group consists of people whose gaming is expected to be related to health problems. Considering that the ICD-11 diagnosis of gaming disorder did not come into effect before 2022 (WHO 2021), the adversity of the participants' gaming habits was determined by their treatment-seeking. The participants were recruited from local hospitals, therapy centers, and other medical institutions, and a call for participation was distributed in the above institutions both in their closed professional networks as well as openly in their social channels. Two such institutions collaborated during the project proposal phase and their networks were applied. As our call was open, we were contacted by participants from outside of the collaborating institutions as well. As an inclusion criterion, the participant had to have expressed treatment-seeking behavior related to gaming problems either personally or as encouraged by someone in their social networks. Any self-reported treatment-seeking of institutionalized help (psychological, psychiatric, therapeutic, etc.) met this condition. As exclusion criteria, we did not include individuals who sought treatment for their play of gambling games, and only non-student participants of 18 years and older were recruited. We did not set demographic criteria, and we highlight that our goal was not to assess the clinical validity of the participants' health problems but rather to understand their experiences. Stopping and minimum: we planned to stop recruitment after having successfully interviewed 15 participants; we set a minimum of five successful interviews as a requirement for carrying out this study to the second phase. Stopping was defined by the limits set by proper transcription, coding, and analysis of the data given the present design. The minimum was defined by the general recommendations of IPA sample sizes and the comparative setting-because we are comparing experiences, less than five participants (even though 
potentially prolific case studies) would not have been enough for our group analysis. Five participants provide enough variation to enable group comparisons.

Group 2. This group consists of people who actively play competitive esports games, while self-reporting no related health problems. Again, we rely on the participants' selfreports in assessing their health; a separate clinical interview design would be needed to properly validate their health statuses. The same applies to 'competitive esports', i.e. it suffices for the participant to identify as a player of such games. These participants were recruited with an open call in local online gaming communities and forums, starting from Kanaliiga (https://kanaliiga.fi) that is an ongoing amateur esports league for working adults in Finland. Additionally, by recommendation of the Finnish Esports Federation (https://seul.fi), we distributed the call to esports teams and other amateur leagues (https://pappaliiga.fi, https://www.gamersunite.fi). Inclusion criteria included more than four daily hours of selfreported esports gaming and having a permanent job. The hourly limit was based on the DSM5 suggestion of 'internet gaming disorder' involving at least 30 weekly hours and previous research reporting 30 weekly gaming hours for those who identify as highly involved players (Vahlo et al. 2018). We do not consider 30 hours to be a universal cutoff, but it appears to represent a culmination point for intensive play in more than one instance. The requirement for a permanent job was applied to better investigate how high gaming involvement is integrated in life situations with regular working hours without expected links to negative health (Quandt, Grueninger \& Wimmer 2009). As with the first group, participants less than 18 years were excluded. We also excluded professional and semi-professional esports players, live-streamers, and others for whom gaming was (part of a) profession; these can be considered special groups that would have further complicated the present setting. In line with our methodology, the focus on non-professional players enabled us to interview a specified (but not too special) gaming group which made analysis coherent-we are not able to generalize across genres, but the material is consistent, with the caveat that esports game design also differs significantly between games. We pursued gender balance but did not set it as a definite requirement because of the expected difficulties related to finding researchconsenting permanently working Finnish adult women who play esports more than four hours daily, within the present time constraints. Stopping and minimum: the stopping point for this group was $n=15$ and the minimum $n=10$. Because participants for this group were expectedly easier to find, we set a higher sample size minimum.

Group 3. This group consists of medical experts such as doctors, nurses, and therapists who have helped at least one individual in their work with gaming-related health problems. Unlike the above two groups, these participants were not interviewed phenomenologically but the function of studying them was to better understand the individuals in Group 1 via a shorter semi-structured questionnaire (https://osf.io/gpme4/). We inquired these experts about how they saw the experiences of people who could belong to Group 1, which provides us a view 'from the outside' and thus enabled triangulating the primary phenomenological data. We separated these Group 3 interviews from our main research question and qualitative hypotheses to exploratively report how the phenomenological findings correspond with what adult expert practitioners (who have treated such experiences) believe or think of them to be. As such, these Group 3 participants were recruited from the same or similar local hospitals, therapy centers, and other medical institutions by snowballing with the help of our institutional collaborators. We pursued gender balance but did not set strict demographic criteria. Stopping and minimum: for each Group 1 participant, we recruited one Group 3 participant. Because the function of Group 3 interviews was to triangulate the findings of 
Group 1, we considered it reasonable to have an equal number of each. There was a possibility that Group 3 experts might be reluctant to consent for an interview due to their role as medical professionals; this could have made recruitment difficult, for which we set a minimum of five interviews so that, in case of recruitment issues in this group, the project could be completed and the main research questions answered even with less data from this section.

\begin{tabular}{|c|c|c|c|c|c|c|}
\hline & Min. & Stop & \multicolumn{2}{|c|}{ Interview and analysis } & \multicolumn{2}{|r|}{ Description } \\
\hline Group 1 & $n=5$ & $n=15$ & \multicolumn{2}{|c|}{$\begin{array}{l}\text { Phenomenological } \\
\text { (POP), IPA }\end{array}$} & \multicolumn{2}{|c|}{$\begin{array}{l}\text { Players with gaming-related health problems, } \\
\text { as defined by treatment-seeking. }\end{array}$} \\
\hline Group 2 & $n=10$ & $n=15$ & \multicolumn{2}{|c|}{$\begin{array}{l}\text { Phenomenological } \\
\text { (POP), IPA }\end{array}$} & \multicolumn{2}{|c|}{$\begin{array}{l}\text { Esports players without gaming-related health } \\
\text { problems, as defined by self-reported health. }\end{array}$} \\
\hline \multirow[t]{2}{*}{ Group 3} & $n=5$ & $n=15$ & \multicolumn{2}{|c|}{$\begin{array}{l}\text { Semi-structured ( } 12 \\
\text { questions), codebook coding }\end{array}$} & \multicolumn{2}{|c|}{$\begin{array}{l}\text { Medical experts who have work experience of } \\
\text { people with gaming-related health problems. }\end{array}$} \\
\hline & Group & \multicolumn{2}{|c|}{ Gender } & $\begin{array}{l}\text { Current weekly } \\
\text { active gaming }\end{array}$ & $\begin{array}{l}\text { Weekly other } \\
\text { gaming activity }\end{array}$ & $\begin{array}{l}\text { The most played } \\
\text { title in history }\end{array}$ \\
\hline Aaron & 1 & \multicolumn{2}{|c|}{$M$} & $56.5 \mathrm{~h}$ & $72 \mathrm{~h}$ & World of Warcraft \\
\hline Bruno & 1 & \multicolumn{2}{|c|}{$M$} & $42.5 h$ & $4 \mathrm{~h}$ & Battlefield 3 \\
\hline Caius & 1 & \multicolumn{2}{|c|}{$M$} & $15 \mathrm{~h}$ & $11 \mathrm{~h}$ & World of Warcraft \\
\hline Dan & 1 & \multicolumn{2}{|c|}{ M } & $25 \mathrm{~h}$ & $30 \mathrm{~h}$ & Stardew Valley \\
\hline Eetu & 1 & \multicolumn{2}{|c|}{ M } & $4.5 \mathrm{~h}$ & $4.5 \mathrm{~h}$ & Age of Empires \\
\hline Fredrika & 1 & \multicolumn{2}{|c|}{$\mathrm{F}$} & $\mathrm{Oh}$ & $5 \mathrm{~h}$ & World of Warcraft \\
\hline Gabriel & 2 & \multicolumn{2}{|c|}{ M } & $35.5 \mathrm{~h}$ & $2 \mathrm{~h}$ & World of Warcraft \\
\hline Heikki & 2 & \multicolumn{2}{|c|}{ M } & $24 h$ & $16.5 \mathrm{~h}$ & World of Tanks \\
\hline Ida & 2 & \multicolumn{2}{|c|}{$F$} & $25 \mathrm{~h}$ & $6 h$ & $P U B G$ \\
\hline Jaakko & 2 & \multicolumn{2}{|c|}{$M$} & & & Rocket League \\
\hline Kalle & 2 & \multicolumn{2}{|c|}{$M$} & $20 \mathrm{~h}$ & $12.5 \mathrm{~h}$ & Magic: The Gathering \\
\hline Lauri & 2 & \multicolumn{2}{|c|}{ M } & $32.5 h$ & $8.5 \mathrm{~h}$ & Counter Strike (many mods) \\
\hline Mikko & 2 & \multicolumn{2}{|c|}{ M } & $51 \mathrm{~h}$ & $18 \mathrm{~h}$ & Rocket League \\
\hline Niina & 2 & \multicolumn{2}{|c|}{$\mathrm{F}$} & $40.5 \mathrm{~h}$ & $2 \mathrm{~h}$ & League of Legends \\
\hline Oona & 2 & \multicolumn{2}{|c|}{$\mathrm{F}$} & $47 \mathrm{~h}$ & $5 \mathrm{~h}$ & Planetside 2 \\
\hline Pasi & 2 & \multicolumn{2}{|c|}{$M$} & & & League of Legends \\
\hline Expert 1 & 3 & \multicolumn{2}{|c|}{$\mathrm{F}$} & \multicolumn{3}{|c|}{ Therapist, has treated approximately 40 treatment-seeking players } \\
\hline Expert 2 & 3 & \multicolumn{2}{|c|}{ M } & \multicolumn{3}{|c|}{ Psychologist, has treated 5-10 treatment-seeking players } \\
\hline Expert 3 & 3 & \multicolumn{2}{|c|}{ M } & \multicolumn{3}{|c|}{ Psychologist, has treated three treatment-seeking players } \\
\hline Expert 4 & 3 & n & & Therapist, has treated & t least 70 treatme & t-seeking players \\
\hline Expert 5 & 3 & n & & Therapist, has treated & $0-25$ treatment $\mathrm{s}$ & eking players \\
\hline Expert 6 & 3 & n & & Psychologist, has treat & d approximately 1 & treatment-seeking players \\
\hline
\end{tabular}

Table 1. Data collection plan (above) and the final sample (below). Weekly gaming time and other gaming activities were inquired by the question "How much time do you spend on the following activities weekly on average?" (translated to English) and six items were provided: computer gaming, console gaming, mobile gaming, watching gaming videos, watching gaming streams, other gaming activities (e.g., modding, forum participation). The first three produced total gaming time; the latter three produced total other gaming time. The average age of Group 1 and Group 2 participants was 30.4, without major differences between the groups. 


\section{Interviews}

Group 1 and Group 2 participants were interviewed with a semi-structured Phenomenology of Play (POP) interview frame (https://osf.io/unmdj/). The development of this frame started in 2020 with a multicultural study $(N=20)$ concerning the gaming experiences of people during the COVID-19 pandemic (Karhulahti et al. 2022a). The feedback and findings gathered in the above enabled us to refine a specialized interview frame (POP) that is designed for mapping out diverse phenomenological dimensions of play. Specifically, the purpose of this interview is to understand how the interviewee experiences gaming and what gaming means to them in their unique biographically shaped sociocultural context. Temporality also has a central role in this interview: distinct parts concern the interviewee's past, present, and future, respectively. Altogether, POP consists of 12 unique thematic sections (lead questions) that have 53 pre-structured follow-up paths. The frame serves as a guide that helps the interviewer to find avenues that are relevant for understanding each participant's experiences as deeply as possible. A translated English version of POP is attached, but the Finnish version was used in this study to enable the participants to express themselves in a native language. One interview was carried out in English, using the English version of POP.

Phenomenological IPA analysis (Smith 1996; Larkin \& Thompson 2012; Smith, Jarman \& Osborn 1999; Smith \& Osborn 2007) serves as a philosophical basis that influenced the development of POP and motivated the understanding of the participants' sensemaking during the interviews as well as in later coding and analysis. The same two researchers (MS and VMK) were present in each interview (recorded audio: $M=112 \mathrm{~min}, S D=28,72-169 \mathrm{~min}$ ). All interviews were conducted remotely (Zoom software). The interviews were recorded by using two voice hardware devices (backup) and afterwards stored as digital audio files in the university's GDPR compliant archive. The audio was transcribed into text word-by-word by researchers and assistants. Personal identifiers were removed from transcripts, which were then submitted for verification to the collaborating Finnish Social Science Data Archive (FSD). FSD will share the data for open scientific use after verification. We removed some parts of the transcripts to protect the participants' privacy-the participants were allowed to review their transcripts and point out parts that they did not wish to share. During this, the participants were also given the chance to comment on our initial interpretations of their experiences; we included these commentaries as part of the data and analyzed them as such. After the transcripts have been successfully stored in the FSD, the audio files will be destroyed. We did not have funds to translate the Finnish transcripts into English. After the interviews, both Group 1 and 2 participants filled out paper-based screening tools (Appendix 2); the scores had no effect on our phenomenological analysis, but we report them in a table as part of our participant descriptions. Detailed demographic data were collected as well; however, to minimize risks, these data are reported selectively and using data abstraction (Karhulahti 2022) to protect the participants.

Group 3 participants were interviewed with a separate semi-structured questionnaire with 12 questions (https://osf.io/gpme4/) that mainly concern their experiences and views regarding treatment-seekers for gaming-related health problems. The same two interviewers carried out these interviews, except in one unexpected instance (only VMK). The length of the recorded audio, on average, was 48 minutes ( $S D=8,41-62 \mathrm{~min}$, all remote). These Group 3 interviews did not directly contribute to the research question but triangulated the phenomenological findings by providing external perspectives to our individual main cases. There is a possibility that Group 3 participants have treated or otherwise aided some of the Group 1 participants; however, we did not inquire about these relationships, with respect to 
the confidential relationships that medical professionals have with those whom they work. In the analysis, we present Group 3 findings as contextualizing data that shed light on the specific sociocultural context in which the main findings take place.

At the time of this study's publication, all Group 1-2 participants were invited for a 1year follow-up interview, following the programmatic preregistration plan. These longitudinal data will be analyzed and reported in a separate publication.

\section{Analysis}

The analysis of Group 1 and Group 2 transcripts followed the established IPA tradition (Smith 1996; Larkin \& Thompson 2012; Smith, Jarman \& Osborn 1999; Smith \& Osborn 2007) with small modification. After these analyses were completed, we moved to analyze Group 3 transcripts. All analyses were carried out in the language of the original transcripts, but thematic and other conceptual entities were developed in English to facilitate reporting. The analytical processes of the first two groups were carried out separately, as follows:

a) We conducted idiographic coding for three interviews and clustered the codes into themes and superordinate themes. The initial structural connections across participants were collected into a table-based iterative document, phenomenological manual.

b) We coded the rest of the interviews one-by-one, with the phenomenological manual as an evolving point of reference.

c) We organized the themes across all (within-group) cases into a refined, visually presented meta-table, supplemented by a summary of each individual case.

The process was a double hermeneutic dialogue in which the researchers interpreted the interviewee who, in turn, interpreted their own experience(s). A more detailed explanation of all three steps is below.

a) After each Group 1 and Group 2 interview, the two interviewing researchers discussed their first interpretations of the meanings and meaning-making expressed by the interviewee. The researchers wrote down notes and this process was repeated for each interview. After all interviews had been carried out, three transcripts were randomly chosen for in-depth idiographic (in vivo inclined) coding from each group. Themes and subordinate themes concerning the meaning and meaning-making in gaming (each participant's unique sociocultural context considered) were interpretively coded by two team members: a researcher who was present in the interview (MS) and a researcher who was not present in the interview (JV). In the end, all coders met to construct a phenomenological manual, which described the relationships between the themes of all three participants in table-based format (Appendix 3, versions 1 and 2). The evolving phenomenological manual documents the development of structural connections; we are not aware of previous IPA studies with this iterative element.

b) All participants were analyzed as individual cases, after which their content was reflected against the phenomenological manual. As earlier, the interviewing researcher (MS) and other researcher (JV) served as coders. Because quantitative coding reliability is suboptimal in IPA, the coders negotiated agreement for each 
transcript (Syed \& Nelson 2015) with a third non-coding researcher (VMK) who close read each transcript and assessed the agreement for each interpreted case. We used Atlas.ti and Excel software in this process (Appendix 3, version 3).

c) Lastly, the researchers collectively assessed structural connections across all within-group participants. The goal was to identify overlap between cases (what experiential themes and meanings are same or similar for two or more individuals) as well as those themes that may be highly important for only one individual. These relationships were integrated into the phenomenological manual, ultimately forming a meta-table (Appendix 3, version 4) that is further explained below by recounting narrative details of the cases. The assessment process was carried out via meetings and remote communication (in a shared online document) between them. After agreement was reached, the fourth team member (not part of interviews or coding) read all transcripts and externally evaluated the validity of the two meta-tables (one for each group). With discrepancies, agreement was negotiated in meetings between all four researchers (Appendix 3, version 4).

After the above three steps had been carried out, the phenomenological macro structures (meta-tables) of both groups were compared to assess their similarities and differences $(\mathrm{H} 1$, $\mathrm{H} 2$ ). This is what we report as results.

After we had finalized the analysis of the two above groups, we applied the final metatable as a codebook (https://osf.io/xev92/) for analyzing the Group 3 medical experts' transcripts. The idea was to see if the experts discuss the experiences and meanings of gaming (disorder) in the same way as they appear in the narratives of treatment-seekers and those players who self-report no related health problems. The codebook was adapted to allow coding alternative beliefs or explanations, too. In other words, for this study, we were only interested in a small part of the medical experts' transcripts. As earlier, the coding was done with Atlas.ti and Excel software by the interviewing researcher (MS) and non-interviewing researcher (RK). We list relevant codes and thematic findings via tables in comparison to Group 1 and Group 2 themes. Ultimately, this section serves as an exploratory contextualizing analysis that helps discussing the individual cases and the research questions concerning them.

\section{Results}

Our analysis produced three subordinate themes for both gaming groups.

Treatment-seekers (Group 1):

i) Escaping and solving difficulties in adolescence by gaming; current play experiences and their associations with self-image shadowed by memories

ii) Struggling with limited means to regulate gaming in a paradoxical situation where it both satisfies basic needs and contradicts personally meaningful obligations

iii) Feeling of guilt or regret for having wasted time on gaming over experiences that would have had "real value" 
i) Gaming is a core component of identity and values

ii) Awareness of gaming having the potential to become excessive, which has produced successful balancing and regulation of play

iii) Gaming is a high-investment meaningful routine

To maximize depth, previous related IPA studies have usually chosen to report only one selected theme (Shinebourne \& Smith 2011a) or provide a comprehensive analysis of one selected participant (Shinebourne \& Smith 2009; Shinebourne \& Smith 2011b). Due to our exceptionally large sample size, we consider it useful to provide an overview of all six themes with examples from multiple participants (see Rodriguez \& Smith 2014). We produce a unique presentation of one participant for each theme. Those readers who wish to investigate any of the cases or theme development/structures in more detail are encouraged to consult the open data and supplementary materials.

\section{Treatment-seeking:}

i) Escaping and solving difficulties in adolescence by gaming; current play experiences and their associations with self-image shadowed by memories

Experienced life problems in childhood or adolescence-bullying, discrimination, and family issues -were present in all treatment-seekers. Many of them explicitly voiced such difficulties to have led them to play videogames excessively at young age. This causality was either direct or indirect: gaming could serve as an immediate escape from these difficulties, or alternatively, as a response to anxiety, depression, and other second-order issues caused by them. There were no consistent patterns regarding how and what the participants playedor whether they played by themselves or together with others-but videogames in general served as a safe place that allowed them feelings of autonomy, belongingness, comfort, and control as a response to otherwise difficult life situations.

Aaron's life changed in the elementary school where he ended up being severely bullied. He never shared these experiences with anyone (until later in his 20s). This left him alone with suppressed feelings in the childhood social world, which he felt incapable of participating. According to him, this caused him a severe depression in adolescence, which, in turn, he started self-treating by gaming.

My depression is connected to gaming very strongly. [As] my depression increased due to bullying [in adolescence], this led me to enjoy gaming more. In game worlds, I could be whoever I wanted to be. It was a means for me to escape the situation and find acceptance. In gaming, I did not have to be afraid of someone bullying me. Or if someone tried, I could just tell them to shut up and ignore or mute them-or if I was the game master, just kick them out... In the real word, I could do none of these things.

These experiences shaped Aaron's years to come. At a critical point in adolescence, World of Warcraft [WoW] became the most central part of his life ("I don't count its play time in hours but years"). The game efficiently satisfied many of his psychosocial needs, due to which he 
never actively sought other life interests. In WoW, Aaron had agency, felt powerful, and was part of a social network that-unlike the world outside-did not bully him. Although the time he invested in the game did not undermine his success in school, it filled very literally all his spare time for eight years straight. When something that he considered important demanded Aaron's time, he could stop playing WoW, but there simply were not many other things that mattered to him. For instance, later in adulthood, Aaron realized that gaming had not left much time for his vocational studies in many years, and he would soon lose the right to study. This realization made him stop gaming entirely for a few months so that he would finish the degree before being kicked out of school.

I remember crying in the bed with my computer at 4AM. [My] mom came to ask me what I was doing and what was wrong; I didn't respond, but just kept typing. I was writing two essays at the same time ... so I did eventually get my degree and for a month after that I had a good feeling, applying for jobs every day. [But] I didn't get even any interviews, so then one day I went to see a friend with my laptop, started playing again and ... that's a blur for next half a year again.

Gaming did not cause Aaron direct harm; rather, the experience of play was one of the few things that made him happy. But this happiness came with a price: gaming remained his sole means for dealing with setbacks in adulthood. Whenever things did not go as planned-e.g., adverse events in family, studies, or work-the pattern that Aaron had learned in childhood reoccurred, as he escaped the situation by immersing himself in gaming for months or years. Today, he is aware of the pattern and the shadow of memories on his gaming ("I flipped again [because of a critical life event] and this was my motivation to play for six months in a row"). When asked about the most important videogame mechanic, Aaron says:

It's that all your decisions and actions have real impact ... whatever you do, it affects the very experience of what you do. That's the most important thing [in games]; that your actions have a meaningful effect.

In Aaron's games (he specifically avoids competition) the play experience provides him a safe place where all actions have meaning, all problems have solutions, and all people are friendly. The phenomenological essence of Aaron's play is a certain "controllability" or "predictability," which manifests in contrast to the uncontrollable and unpredictable events of everyday life in the past, and sometimes, the present too.

\section{Treatment-seeking:}

\section{ii) Struggling with limited means to regulate gaming in a paradoxical situation where it both satisfies basic needs and contradicts personally meaningful obligations}

In later childhood, Fredrika and her family moved to a new city. She became lonely in a new school where other girls started bullying the "newcomer." As a response, she started gaming more and in later adolescence found a male gaming community around WoW in particular. Unlike the girl groups from which she had been left out, the boys and men welcomed her as one of the few women who had the motivation and skills to keep up with the rapidly changing, mechanically demanding online game. Over the years, Fredrika grew up in the community 
and found a partner from there as well. Her school performance was good and later she successfully worked in diverse jobs, but she and her partner spent all their shared time in WoW. At first, this was a meaningful part of their lives-their gaming was heavily competitive and successfully so-but as a decade went by, this meaningfulness vanished.

One day I just started to feel sick. I had to leave and go find myself again ... I've only now come to understand what [those years] cost me, but I also know that I couldn't have made other choices at the time ... I needed a community where I could earn my place by perfection [in the game], and that's my wound ... I tried to turn my childhood memories around by redeeming a place to belong.

While gaming had been an effective way out of the loneliness and bullying that Fredrika suffered in childhood, gaming also turned into a perfectionist enterprise for her that she could not simply replace with something else. For a decade, her entire social world was in the game-including her partner-and to maintain her position in that social world, she had to perform well and keep up with the game's competitive updates. However, even after finally leaving WoW and going for a "search of a new self," she finds it difficult to have gaming as part of her life in any form.

Currently I don't play at all because when I do it just gets out of hands, and I cannot find a game that would be a massive dopamine hook so that it would allow me to play short periods ... every game is just too dangerous to me. It's terrible to realize that it's me who abuses the games, but then again, they're also designed to be abused. I don't want to do it for myself, I rather want something else [with my life]. I hope I'll never return to [WoW], ever ... I just cannot enjoy gaming neutrally, without paying the fee that is too high. That's why l've quit totally, but am still hoping to play again, one day.

On one hand, Fredrika would like to find a game that is a "massive dopamine hook." She believes such game could give her positive feelings fast without keeping her stuck for those 12-hour periods that do not leave her time for "life." At the same time, she also perceives the problem to be in her own "abuse" of the games, which leaves her in a paradoxical situation with a solution to make a change in how she plays, but that is just not possible. Therefore, she rather chooses not to play at all. But that is not unproblematic either, as the decade of playing WoW has significantly contributed to how she has come to perceive the world in general. Her agency in everyday interactions-mediated by her social networks, language, and skills-still involve WoW even though she does not play it anymore.

[A friend] just asked me to come and play again, as there was an event that gives a free mount [virtual game item]. Instantly I started fearing that if I wouldn't go, I might lose the rare mount and I would regret later, it's only a one-time event. And every time I experience that same disappointment, fear, regret-all of it, I realize it's so right for me to not play anymore... my brains have somehow been adjusted to still want those things that I don't want anymore. I mean, think about it: I've already decided to never play again let alone collect any mounts, and then I hear that I might lose a mount that I'd never ever want, and I will instantly start wanting it and feeling disappointedfearing for the loss of something that I don't even want [she emphasizes]. 
Our treatment-seekers described gaming to satisfy their psychological needs, and the efficacy thereof was also the very reason why they found it difficult or impossible to control gaming. Over years or decades, they had learned to utilize gaming effectively to make life enjoyable and meaningful, but as they grew older with evolving perceptions of self and value, replacing gaming with something else-despite trying to do so-was difficult. To some degree, videogames had come to mediate how they interacted with the everyday world.

\section{Treatment-seeking:}

\section{iii) Feeling of guilt or regret for having wasted time on gaming over experiences that would have had "real value"}

Eetu was bullied throughout the entire elementary school, for seven years. During those years, he was lonely and felt as an outsider. All his spare time was spent on playing various single player games at home. Eetu's parents would encourage him to do other things but did not limit gaming time, as the school grades were good ("what else could I focus on during schooldays if not studying")-it never occurred to them that something was not right. Eventually, his parents got divorced. Eetu was diagnosed with anxiety, which he did not want to be treated (medically); rather, he escaped such feelings by playing videogames, in which everything was in his control.

The videogames I like most are those with a mouse as control [stresses the English word], it's an instrument for controlling and pointing things, like "you go there" and "you do this thing here" ... these are mainly strategy games, like you select units and order them to "walk there" and "attack there" or choose building tasks for them to do from a menu ... and they build it where you tell them to. [Those] games that had readymade slots where buildings would surface automatically after clicking, they just didn't feel good. I specifically liked those games where I could choose someone and say, "you there, go do this thing."

Unlike Aaron and Fredrika, Eetu did not play online games, as there he would lack control and be vulnerable to other people's actions. Single player strategy games provided him the autonomy that everyday life did not ("they were a secured source of pleasure without the punishment of failure"). Gaming thus remained a means for him to cope with the uncertain and unfriendly world around him; however, having played 10-12 hours daily for years eventually made him reflect. In his late 20 s, during higher education studies, Eetu failed to return his thesis year after year because of not being able to stop gaming. This led him to recognize gaming as a problem for him. He tried to quit, but found it impossible.

When I play, it's like all the cells in my body scream "this is what we were made for!" It is such a pleasurable, activating and stimulating feeling; a bodily experience-not philosophical nor related to my identity-but that's just how it feels when I play. And that's very sad.

As Eetu found a partner, she advised him to seek treatment and they started developing regulation strategies together. This meant that Eetu gave away all administration rights to all the computers (being unable to install any games), and installed a program called Cold Turkey 
Blocker that denies access to browser gaming as well as related videos and websites ("I can easily spend a whole day watching the game I want to play from YouTube or Twitch"). Now he feels that gaming is in control, but at the same time, also being afraid that one day he might relapse. When asked how he would feel if gaming were taken permanently out of his life, he responds:

Eetu: That would be a relief. I could stop struggling, and I wouldn't have to be afraid anymore ... it would give me [and my close ones] freedom. I am now living a life where I already pretend things are like that, but I'm also aware that I cannot ever play again, and this makes me feel that I'm permanently ruined as a human. Although I can extinguish my need, my brains will always be ready to flame up again and reactivate the behaviour just like before.

VMK: How would [removing gaming permanently] affect your life in the long run?

Eetu: It would help me be a good husband, father, and an employee ... Because then I would not have to be afraid that, one day, everything might go all the way to hell again.

For Eetu, the very nature of the problem with gaming is that, as he puts it, "I always wanted to do other things but gaming." He tells about having started hobbies and taken various responsibilities in organizations, but in all such tasks he failed because gaming overtook his life. Gradually, he thus developed an understanding of the paradox between what he wanted and what he did; the older he grew, the more he became aware that gaming conflicted with what he really wanted and valued.

All gaming is a waste of time when you compare it with the rest of life. If there's anything you learn in a game-something you can apply elsewhere-you can learn the same thing much faster outside the game. [There are] few other activities that are as useless as gaming in terms of what skills and knowledge you learn in it ... Gaming is just gaming, [it] requires developing many skills but they are all gaming-specific skills.

The feelings of guilt or regret for having spent so much time on gaming - which was perceived inferior to the achievements and "real values" of life-forms our third subordinate theme of treatment-seekers. In different ways, they all had become aware that gaming was not what they wanted to do to the extent that they did; gaming was not (or no more) among their hierarchy of values, but changing the daily behaviors that they had developed over years and decades was not, for varying (partly unknown for us) reasons, easy to implement in practice.

\section{Esports players:}

\section{i) Gaming is a core component of identity and values}

Niina's gaming started as a child with her father who travelled a lot because of work. She always looked forward to him returning so that they could play videogames together. Henceforth, gaming sticked with her as a beloved hobby. She recalls beating her older brother in a game one day and him admitting defeat, which was an empowering experience and increased her self-confidence. Niina's parents also encouraged her to try many different hobbies and sports such as arts, crafts, horse riding, and swimming. Her memories of 
childhood are highly positive, but at the same time, she also struggled to meet the gender expectations set by the society around her.

My social circle was soon mostly boys, as I realized they have much more in common with me than girls do. I wasn't interested in fashion or make-up at all, but more like cars, gaming, and tech. This moved me toward the gaming world especially in the gymnasium where I was only hanging out with boys who played games like Dota and LoL [League of Legends] ... I rather spend my time with people who have the same preferences and interests, but of course it would've been nice to have another girl there, you know, to talk about women's things and have someone who understands.

In many ways, the beginning of Niina's story is identical to that of Fredrika's. In early adolescence, they both had to construct an identity in a social world where girls did not (and were not expected to) play videogames. As Niina grew older, the people she interacted with (from relatives and peers to even teachers) kept telling her that gaming is not for girls, and this critique later extended also to her other interests when she decided to study programming ("even my parents told me that I shouldn't choose such male dominated career and I'd never be able to succeed there"). The conflict was most prominent in the classroom where she was also bullied, and this one day led Niina to cut her long hair off and, in her own words, "start my life as a boy." The act reflects her exceptional resilience-instead of escaping from or submitting to the environment, she always chose to fight it.

I've always had to fight. I do recall thinking like, what if I'd just become a hairdresser or something like that ... but then again, I'm somehow competitive, so I rather just choose otherwise and tell myself "I'll show 'em." Whenever someone tells me I cannot do it, I'll just say "we'll see about that." [That's] probably also why I like gaming so much. People told me I cannot play because I'm a girl, so I feel like I need to fight, not only for myself but for other women too.

Although Niina plays esports several hours daily, she is clear about not playing competitively against others. When asked what gaming means to her, she says play is a means to express, challenge, and realize herself [toteuttaa]. This challenge must be controlled, however, due to which she does not play competitive esport modes where her performance would be ranked against other people. She wants to face challenge, but only among and with the people she knows, without external competitive pressure. Speaking of LoL, which has been her main game now for the last five years, she explains:

being ranked would be too stressful ... I'm a little uncertain if I would do well there, [albeit] I'm tempted to try just to know my skill level ... but I also want to protect myself from the obsession of needing to prove myself by a rank.

Unlike Fredrika earlier, Niina has explicitly chosen not to start competing, as she knows it would lead to obsessive frustration and the need to show herself and others that "I can." She rather plays in modes where losses and wins are not measured or ranked into a hierarchy. Because she had been fighting for the right to have an identity that did not fit the norms of the society throughout her life, in this case, she chooses not to fight. While playing esports games is a core part of her identity, she is also afraid that taking "one more fight" could turn 
it into an obsessive enterprise that harms her wellbeing. This is one of the many ways in which she consciously regulates her gaming daily, in addition to having rules for stopping and doing other things when mental, physical, or other alarms tell her to do so. As such, gaming remains a foundation of Niina's identity and life that she would "never like to quit," as quitting, according to her, would directly lead to anxiety and depression because "such a large part of who I am would be gone."

\section{Esports players:}

\section{ii) Awareness of gaming having the potential to become excessive, which has produced successful balancing and regulation of play}

Oona grew up in a small town in a family with three siblings. She describes her childhood as stable, with the same hobbies and friends following her throughout schoolyears. Unlike Fredrika and Niina, she soon found other girls whom she could play with, but single player games remained her main type of gaming until later adulthood. Oona has always been extremely active-she tried dozens of different hobbies and sports in adolescence, and eventually ended up also in politics, which she still does as an extra volunteer job next to her fulltime employment. These days, she is extremely busy, but at the end of each day, she feels that gaming is a means for her to "reset" and recover from hectic schedules. The game she plays most is Planetside 2, a competitive first-person shooter where large teams work together and combat against other teams.

I work from Monday to Friday and my day starts at 08:00 or 09:00 and ends between 16-18:00. I'm at home around half seven, and after I've heated up the food, I sit on the computer. I prepare the food for the whole week on Sundays, so I can just heat it up. I stop playing around midnight. It's a reset for my brain when I can just shoot in Planetside [2] and I don't need to think because it's automated and easy-and it's automated because I've played so much ... sometimes I don't have to play or even think about it; my life is so hectic that, for me, gaming is specifically a way of calming down and doing something pleasurable.

Oona is not interested in the competitive events of Planetside 2, as her gaming is not "goaloriented [and she has] no time nor interest for serious competition." Yet she does play several hours daily and carefully reads every update and patch for the game-just like Niina, she is highly invested but does not have the motivation to "take the fight" in seriously competitive play, as her life is already full of work that satisfies her achievement needs. This is partially captured in how she compares her other hobby, politics, to gaming: "I play so that I can reset my brain; I do politics so that I can change the world." For Oona, gaming fills a significant place in her identity, and it is something that she loves to do several hours every day. But it is also just one part of her identity. When asked if she feels gaming is taking too much of her scarce time, she responds:

No, I don't have the time to play too much. I wish I had, so that I could sometimes play as much as I want. But as an adult at work, with commitments in organizations and many other places, and tight schedules pushing with also trying to maintain friendships, family, and other relationships, I simply don't have time to play too much. 
Somehow, Oona has built a clear hierarchy of values and a list of priorities, in which gaming holds strong positions but does not interfere with the rest. She believes that a clear sign of playing too much would be the neglection of her social relationships, yet because almost all of her close social relationships (family, friends, partner) share the interest in gaming or at least spending time online (Discord), it would rather be the other way around: reducing gaming time could lead her to neglect important social networks. Due to her busy life, Oona is constantly reflecting on how she uses her time, but a conclusion of that reflection is simply that gaming keeps providing her exactly what she needs to keep the life in balance.

At one point I was unemployed and [started] feeling like I might be playing too much. But then I realized that I hadn't neglected any of my wellbeing. I didn't skip social events, meals, or sleep ... the feeling surfaced just because I was used to living a hectic life and suddenly, when I didn't have it anymore and played 10+ hours daily, it made sense to ask "is it ok for an adult to play so much?" ... but I knew there was a job starting soon [so] I though "this is just a phase, enjoy it now, you'll soon be grinding in the workplace again."

Just like our treatment-seekers, Oona had played (and was still playing) a lot and believed that, in excess, it could negatively affect her wellbeing. But throughout her life, she had always had many parallel life goals, and she had always worked to build a balance between them. Even though she routinely played the entire evening after her workdays, she also had a clear system to eat before starting to play and shut the computer by midnight, thus ensuring that gaming would not lead to skipping meals or lack of sleep. Like other things in her life, gaming was strictly reflected, regulated, and fitted to a highly scheduled life.

\section{Esports players:}

\section{iii) Gaming is a high-investment meaningful routine}

Gaming has been the most important activity in Kalle's life since childhood. One of his clear childhood memories is a teacher telling his mother to buy a better gaming computer for him. Kalle performed extremely well in school but did not have many friends, the latter of which he believes is because of his "personality type" and the fact that gaming, his core hobby, was stigmatized at the time. His life changed in later adolescence, as he found the (analog) card game Magic: The Gathering [MTG]. Kalle soon became part of the community around the game and found several new friends who enjoyed competing in tournaments. He specifically recalls a local tournament, which changed his life.

We drove there 100 kilometers in a bus with friend [and] as I walked there, into the event, it was as close to a religious experience as I have ever been. Like, there were 200 people playing this game ... it was just ... just something unbelievable. And I felt ... felt like ... I had finally arrived home. After that day, I played the game [MTG] seriously for a decade, travelling tournaments with my friends around Europe and the US, and most of my best friends as well as the current job derive from that time. 
Kalle's work is related to gaming, being responsible of data analysis in sales. After the gymnasium, he studied in two higher education institutions but never finished either degree. He rather preferred to invest his time on learning about different games, which eventually opened him occupational opportunities. Kalle's life has always been tangled around gaming in all possible ways, and his partner, for almost two decades, plays games actively too. Kalle notes that working is part of his life only because he needs the money-his life is meaningful for other reasons. Currently, most of his time goes to Counter Strike [CS], which he started playing seriously after quitting MTG. He always plays to compete, and if the games do not provide him competitive satisfaction, he finds a new game.

I recall the specific moment when I realized I don't want to play MTG anymore. I was in an [international tournament] with friends, and the trip was good, and games went ok, but then I just noticed that losing didn't matter to me. And in that very moment I had the experience-like, if I don't get frustrated by a loss it means that I don't want to win enough. I don't enjoy playing just because of playing, so if my competitive interest for this game is dying, why would I be playing it at all. That was the moment. And after that moment I quit playing MTG permanently.

Currently, playing CS provides Kalle with the same competitive challenge that MTG gave him for more than a decade. He has already reached the highest possible online rank in the game several times, but also knows that he will never (want to) be a professional. Rather, Kalle competes with his amateur team and friends on their "subjectively meaningful" level-what is left is the essence of competition as such, against other amateurs. The important thing is that he can and must do his best to win before, during, and after the tournaments they participate. It is an all-encompassing and continuous experience of learning and improving, which he enjoys for its own sake. Regarding the best moment in Kalle's gaming experiences, he struggled to answer because "everything is blended into one." Yet it is by far the most important activity in his life.

If I couldn't play electronic games anymore, I would just start playing more board and card games. And if I couldn't play board and card games, I'd likely be in a very good shape [implying he would start playing more sports games]. As an adult, I've specifically arranged my life so that gaming isn't a problem for me. I can easily be without gaming when there are things to do. But practically, my life is organized so that I can play as much as I want.

The holistic degree to which Kalle enjoys and invests in competitive gaming was exceptional, also in the context of our active sample. Although all our esports participants played more than four hours daily, for Kalle, "gaming is life." Importantly, it did not matter much to him which type of gaming served as the medium enabling competition; however, computer-based gaming was the most accessible option, as it did not entail travel and his old sports injuries did not limit participation.

\section{Meanings by Experts}

The six subordinate themes characterizing treatment-seekers and esports amateurs, respectively, could be identified also in the experts' experiences regarding gaming-related 
treatment seeking-yet in different ways and sometimes with only a thin link. Because our subordinate themes derive from in-depth analysis of specific cases, it was often difficult to interpret whether the experts' descriptions match the players' experiences to a sufficient degree. Examples of case descriptions that overlap with the above six subordinate themes are presented in Table 2.

\begin{tabular}{|c|c|c|}
\hline & Subordinate themes in treatment-seekers & Subordinate themes in esports players \\
\hline i & $\begin{array}{l}\text { "...for the majority, gaming is used as medicine for } \\
\text { different emotional states ... that's associated with } \\
\text { the positive feelings that go way back in time" [E5] } \\
\text { "... they have already been outcast or excluded from } \\
\text { the society at young age" [E1] }\end{array}$ & $\begin{array}{l}\text { “...it's also a long-term hobby that didn't always } \\
\text { involve problems and it's difficult to believe } \\
\text { that it wouldn't also be highly meaningful" [E5] } \\
\text { "... they've always been gaming, even } \\
\text { committed [but] later grown up with new } \\
\text { responsibilities, and less time for gaming" [E1] }\end{array}$ \\
\hline ii & $\begin{array}{l}\text { "... there can be a certain defensiveness ... like they } \\
\text { want to believe it isn't problematic [but it is]" [E6] } \\
\text { "For those working [it is] typically a job without clear } \\
\text { workhours and the line between work and non-work } \\
\text { is thin; if there's then a highly interesting activity } \\
\text { available [gaming], of course it will disturb" [E5] }\end{array}$ & $\begin{array}{l}\text { “...the person was able to perform when their } \\
\text { child [was present] but alone just played and } \\
\text { every plan was left undone [until] making a } \\
\text { decision to quit [which] fixed their life." [E2] } \\
\text { "...one doesn't even have to reduce gaming } \\
\text { [but] rather regain control of life and activities } \\
\text { [which] then changes gaming too" [E4] }\end{array}$ \\
\hline iii & $\begin{array}{l}\text { "Some simply state that [gaming] has become too } \\
\text { excessive and replaced other things" [E2] } \\
\text { "Usually, it's part of a bigger problem set, involving } \\
\text { dissatisfaction with one's own life situation ... I don't } \\
\text { recall any case where gaming is the only or even main } \\
\text { problem [but] also the gaming amounts are of course } \\
\text { way too large against their responsibilities." [E5] }\end{array}$ & $\begin{array}{l}\text { "What makes gaming so-called addictive [is] } \\
\text { agency and the complex meanings it involves; } \\
\text { not least the rich cultures and communities, } \\
\text { which are way more significant compared to } \\
\text { passive spectatorship" [E1] } \\
\text { "You must invest and immerse in it; you cannot } \\
\text { switch to dabbling after playing so long" [E4] }\end{array}$ \\
\hline
\end{tabular}

Table 2. Experts' descriptions of treatment-seekers, with which they have worked, related to our six subordinate themes. Note how the experts' descriptions also overlap with the themes that we identified from esports players.

In addition to the above, we also identified distinct themes of experience and meaning. Many of these themes strongly overlap with our six subordinate themes; or rather, represent small elements in them. As the experts' views were evolved and refined over wide-ranging histories of work, they represented common, "surface-level" themes across cases rather than deep, indepth themes of individual cases. These themes are presented in Table 3.

\begin{tabular}{|l|l|}
\hline Meaning of experience & \multicolumn{1}{c|}{ Reference } \\
\hline Stress relief & $\begin{array}{l}\text { "It's stress relief, when there are no resources left and one is the most tired, } \\
\text { that's when they need to check the notifications from the game and the urge [for } \\
\text { other gaming-related things] sparks" [E1] }\end{array}$ \\
\hline $\begin{array}{l}\text { Accomplishment, } \\
\text { belonging, feeling valued }\end{array}$ & $\begin{array}{l}\text { "If you have a community that values you and your effort, well, that's naturally } \\
\text { very narcotic ... the feelings to belong, and to be good at something." [E1] }\end{array}$ \\
\hline Accomplishment & $\begin{array}{l}\text { "If a human being has limited mental or other resources, for any reason, } \\
\text { [gaming] can give the feeling of having accomplished something." [E2] }\end{array}$ \\
\hline Belonging, escape & $\begin{array}{l}\text { "It can be therapeutic, like escaping from an anxious situation [by] playing } \\
\text { compulsively or reflexively ... there can also be a social element, which gives the } \\
\text { feeling of doing something together" [E2] }\end{array}$ \\
\hline $\begin{array}{l}\text { Belonging, escape, a } \\
\text { means to focus }\end{array}$ & $\begin{array}{l}\text { "It's mostly an escape from the everyday, and a means to really focus on an } \\
\text { activity, which then keep one going ... then again, there's also the social aspect } \\
\text { [which] can be meaningful as allowing one to feel participation" [E3] }\end{array}$ \\
\hline Escape, living in the past & $\begin{array}{l}\text { "Many are (re)living nostalgic youth, the wonderful early moments that gaming } \\
\text { brough them ... but for others it's a place to escape challenges and problems in } \\
\text { life, or just avoid them temporarily." [E4] }\end{array}$ \\
\hline
\end{tabular}




\begin{tabular}{|l|l|}
\hline Escape & "Those whose gaming is problematic, well, often it's a matter of escapism." [E5] \\
\hline $\begin{array}{l}\text { Accomplishment, } \\
\text { belonging, control }\end{array}$ & $\begin{array}{l}\text { "They seek a world that they can control [which] is something they do not have } \\
\text { otherwise ... but it can also be about feelings of success, and social contacts. And } \\
\text { of course, those two things are connected." [E5] }\end{array}$ \\
\hline $\begin{array}{l}\text { Comfort, escape, safety, } \\
\text { shield }\end{array}$ & $\begin{array}{l}\text { "Playing for escape is a rather common theme for our players ... it's sort of a safe } \\
\text { place, a shield. Some have even said that, without that shield, they would have } \\
\text { ended their lives already ... The meaning is in comfort and safety" [E6] }\end{array}$ \\
\hline $\begin{array}{l}\text { Accomplishment, } \\
\text { belonging }\end{array}$ & $\begin{array}{l}\text { "Often there's also the social contact ... there can be an element of self- } \\
\text { development or improvement, but the social part remains most central." [E6] }\end{array}$ \\
\hline
\end{tabular}

Table 3. Experts' descriptions of treatment-seekers' meaning of experience.

Considering the occasionally evident overlap between the themes representing our participants' experiences and our experts' terminology, we note the possibility of various looping effects; for instance, treatment processes and related materials having affected how the participants internalize and verbalize their experiences (see e.g., Hacking 2004; Matthews et al. 2017). At the same time, similar looping effects-with entirely different concepts and terms internalized-may have occurred in our esports players' social environments, too. Because our study was not planned to address the issue, we leave this note without further development and encourage future research to investigate these possible effects explicitly in carefully planned study designs.

\section{Discussion}

To our knowledge, this study has been the first one trying to investigate the phenomenology of gaming disorder with actual adult treatment-seekers, and more specifically, how it differs from the experiences of intensively gaming esports players who do not consider the activity problematic for them. Our in-depth analysis suggested three unique themes for each group, and their differences can be summarized as follows.

For treatment-seekers, the experience of disorder derives from gaming interfering with self-what one wants to be, do, and have throughout life. For esports players, the experience of balance derives from gaming being integrated into self-what one wants to be, do, and have throughout life.

Although we did not set hypotheses for testing, it is worth briefly discussing how the study has updated our beliefs. First, following our expectations (QH1), we did find some treatmentseekers' experiences to occur in the context of neglected needs or responsibilities, lack of control, and social difficulties. However, there were no consistent patterns regarding what needs/responsibilities were neglected and how, and while social difficulties (like loneliness) were present in many early life phases, the participants did not generally consider their present social lives deficient. Our study was not biographical, and we did not explicitly code causalities from the data, yet it became evident that social difficulties and traumatic social experiences in childhood-especially bullying (five out of six cases)-had significantly affected the participants and their current gaming behavior with derived experiences.

Many of the players in the second group had similar adverse childhood experiences, but they had somehow acquired networks of resilience that prevented them from "falling" and they 
rather utilized gaming protectively, as part of a stable identity. The same applied to "loss of control," which was present in both groups in multiple forms; for instance, as having lost control in daily life (educational, occupational, and social domains) and having lost control over gaming (for several years, months, or in a moment of a single session). Almost all participants admitted having lost control sometimes. As a key difference, the esports players had succeeded in developing self-regulation routines that simply worked for them. It is left for future studies to investigate the (expectedly numerous) factors that allow intensively gaming individuals to self-regulate efficiently (but see also Sripada, 2022).

We also expected gaming to be meaningful for both groups, in terms of self-development and social value $(\mathrm{QH} 2)$. This was the case for many esports players, but more complex patterns characterized the treatment-seekers. Our interpretation of the present data is that gaming had been meaningful as a self-development activity for many treatment-seekers, too, but that meaning had also started to inflate or vanish (usually after tens of thousands of gaming hours). At the same time, those treatment-seekers who had been playing multiplayer games struggled to let go of the social networks on which their entire social lives had been built over the years. This was one of the conflicts that defined their relationship with gaming: feeling that other life goals were more meaningful than gaming, yet it being difficult to pursue those other life goals without sacrificing the psychosocial benefits of gaming. Even if gaming was meaningful, it was not meaningful enough to justify-for them-such significant investments of time. Below, we discuss the themes of both groups in pairs.

\section{Treatment-seekers}

Escaping and solving difficulties in adolescence by gaming; current play experience and their associations with selfimage shadowed by memories

\section{Esports amateurs}

\author{
Gaming is a core component of identity \\ and values
}

Childhood and adolescence are critical periods for neurodevelopment (Chambers et al. 2003), and adverse childhood experiences such as bullying are one of the strongest predictors of future problems (e.g., Kumpulainen 2008; see Schimmenti et al. 2014). Although our study was not planned to address this issue explicitly, we found the treatment-seekers' gaming experiences to be strongly connected to their childhood traumas and bullying in particular. This is consistent, for instance, with the early theory by Jacobs (1986), according to whom addictions are "an attempt to correct a chronic preexisting stress condition" (p. 20), and they develop around opportunities "to escape even briefly from the unpleasant realities of everyday life to a world apart wherein [one] may actively live out their fantasies of personal success and of social acceptance" (p. 21). Phenomenologically, in our data, this manifested as a constant need to be in control and safe-and gaming provided exactly these feelings during and after adolescence. Over the years, gaming then evolved into an essential source of daily psychosocial resources, which the treatment-seekers found difficult to live without.

In an aptly named study "How there can be a mental disorder in a normal brain," Wakefield (2020) gives a useful analogy of goslings (baby geese) that famously "imprint" on the first creature they see after hatching. As Wakefield points out, this entirely healthy and normal biological design may also turn against the goslings if they are unlucky and the first thing they see is not their mother but, say, a fox: 
Once imprinted, the gosling then has an irresistible and irreversible desire to stay close to and follow the represented creature... One can identify what has gone wrong only by going beyond brain descriptions and referring to meanings (i.e., what the gosling's brain-stored image in fact represents) ... The point is that there is nothing wrong with the gosling at the brain-descriptive level. The gosling's problem is a matter not of brain functioning per se but of the reference or meaning (p. 6-7, emphasis added)

There are similarities between the treatment-seekers in our study and the goslings who, by the stroke of bad luck, construct "wrong" meanings (more about "wrongness" below). Due to the circumstances, which the treatment-seekers had little control over, they ended up building meaning and value networks largely around gaming at young age. As these networks were left narrow and circular, their capacity to seek new meanings and values tailed off which, in turn, further increased the significance of gaming at the cost of everything else. This can be further explained by enactivist phenomenological perspectives, according to which our bodily and historical involvement with the world, over the lifetime, come to define how and what things mean to us. As Noë (2009) explains:

What gives my thoughts their content is my involvement with the world ... Meaning is relational. And the relation itself thanks to which our thoughts and ideas and images are directed to events, people, and problems in the world is the fact of our being embedded in and our dynamic interaction with the things around us. (p. 164)

If a person's life has consisted almost exclusively of interpreting the world with and through gaming by tens of thousands of hours of play-originally as a reaction to bullying or similar events-it is but natural that their constructions of meaning and value outside gaming, too, are shaped by that history. Nonetheless, as our esports players' experiences imply, this shaping does not affect a person's thriving or wellbeing negatively by default: whereas the treatment-seekers felt gaming to interfere with their identities, gaming kept contributing to the esports players' identity projects. In fact, most of the former were currently going through a major reconstruction of identity, like previous clinical research has shown to happen in the recovery phase of diverse problematic behaviors (Koski-Jännes 2002). Had we interviewed the treatment-seekers five years earlier, would their experiences be like those of the esports players? And will some of the esports players be going through a similar crisis of identity in five years from now? Longitudinal phenomenological studies (including the follow-up of the present study) will hopefully yield answers to these questions soon, but the fundamental group differences in identity, meaning, and value structures lead us to hypothesize the null.

Ultimately, we cannot explain how people in almost identical conditions end up building radically different identities and pursuing different paths. Some of our esports players seemed to have equally negative childhood experiences that led them to play videogames excessively in adolescence, yet they still managed to maintain interest in many life activities, social relationships, and build successful careers without feeling their gaming experiences to interfere with the above. The answer to this mystery is beyond the data and methods of the present study, but regarding the mapping of potential factors, we refer readers to the emerging philosophies of complex science (e.g., Mitchell 2012; Olthof et al. 2022) and the 
preliminary attempts to model such complexities in addiction research (e.g., Blaszczynski \& Nower 2002; Brand et al. 2019; West \& Brown 2013; see also Clark et al. 2022).

\section{Treatment-seekers}

Struggling with limited means to regulate gaming in a paradoxical situation where it both satisfies basic needs and contradicts personally meaningful obligations

\section{Esports amateurs}

Awareness of gaming having the potential to become excessive, which has produced successful balancing and regulation of play

Over the past decades, one of the key meta-conceptual questions in addiction research has been: what if a person chooses to live by excessively undertaking substances or behaviors that harm their health (e.g., Foddy \& Savulescu 2010; Skog 2000)? Should the person still be considered "addicted" if a life with "addiction" is the life they prefer? Although such questions are increasingly relevant with the new addictive behaviors diagnoses in the ICD-11, for our treatment-seekers these were not key issues because they all explicitly wanted to play less or quit entirely; they just had trouble doing so. The esports players too voiced either previous or continuous efforts to regulate their gaming time-but without trouble succeeding (with equipment like alarm clocks and schedules reminding them to not play too much). Only the treatment-seekers lived in a paradox where their need to play clashed with the need to play less. Their reason for seeking treatment was the self-defined need to learn better regulation strategies and to build up resilience in relation to their learned patterns.

A review of the qualitative literature on health behavior changes suggests that such changes are first and foremost prompted by "an intolerable conflict with personal values and goals" (Kearney \& O'sullivan 2003). As discussed in the previous section, our treatment-seekers' need to play less was strongly related to their realization of gaming conflicting with their current identities or values; however, it is important to highlight the variety of these experiences.

- In the case of Aaron, gaming served as his core means to cope with setbacks, while it also remained as an important medium for social interaction that allowed him to stay in contact with close friends.

- For Fredrika, gaming was not a means for coping, but her entire social network had been built around videogames, which made her spend time and seek meaningful experiences in gaming despite not wanting to do so.

- While Eetu played only single-player games, he considered gaming to satisfy a strong (corporeal) need for autonomy and success, which he also sought at work but had not managed to find so far.

- Dan identifies as an introvert and reflects (single player) gaming through neurodiversity. He constantly needs to do and cannot stand any boredom. Gaming keeps him calm and well, but occasionally leads to "hyperfixation", i.e. he may not be able to stop for months.

- In contrast to Dan, Bruno is social and never felt "fitting in" with the silent Finnish culture. While gaming remains his main medium to experience art, express himself, and belong to meaningful communities, he also feels it may not allow him to "proceed in life."

- Caius describes being "stuck" with the community of his online game, which has made it difficult for him to quit so far. He really wants to quit and is now trying to establish "real life" connections with people and seek similar "feels" outside gaming. 
Although we did not code the needs that gaming satisfied for the participants nor the needs that were left unsatisfied, these are worth discussing. For instance, of the 16 human needs identified by Reiss (2012), in our data, we can see gaming contributing at last to acceptance, curiosity, family, honor, independence, order, power, romance, saving, social contact, status, tranquility, and vengeance. In the same way, for some participants, gaming evidently contributed to Maslow's (1943) basic needs (safety, love, esteem, and self-actualization) as well as the basic psychological needs recognized by self-determination theory (Ryan \& Deci 2017; autonomy, competence, relatedness). The differences between individuals were radical, nevertheless. Whereas some treatment-seekers played team-based online games whose intense social nature defined both the experienced benefits and harms, others played single player games that provided exactly the opposite, solitary stimulus beyond all social interaction with others. Likewise, whereas some treatment-seekers had been swept away with the intense competition provided by the games, others explicitly sought games that represented safe places where competition is not present.

In sum, we can see how specific videogame designs "match" with the specific difficulties and needs that people have in their diverse life situations. In some cases, because of multiple interacting factors, these player-gaming relationships may help people to thrive in their lives-but also develop into problematic patterns. For instance, certain videogame designs may negatively interact with trauma-coping (such as overcoming bullying experiences), and specific designs may negatively affect certain neurodevelopmental types. At the same time, our data still come from a single country and culture, and we are left wondering what other ways of "having problems with gaming" might manifest elsewhere. Partanen, one of the pioneering Finnish researchers of substance use, pointed out these challenges in the 1990s regarding the WHO's attempts to create universal criteria and instruments for alcohol and drug conditions:

[the] fundamental axiom is that the "disorders" are essentially the same everywhere, only their prevalence varies. The line of action is to continue to spread the message to the rest of the world. [But] the very logic of substance use disorders in particular cultures derives from the socio-economic and cultural matrix of the whole society. There is, of course, a reason why alcohol and drug [researchers] shirk adequate analysis ... [it] would reveal that in practice there is very little they can do to help to deal with substance use disorders. (Partanen 1996, 225-226)

We must repeat that the problematic relationships our treatment-seekers had with gaming were strongly connected to their distinct sociocultural roles, which may differ between countries and cultures (see Kleinman 1988; Singer 2002). Regardless, our findings are perhaps the strongest evidence so far to demonstrate the specificity and diversity of problems related to gaming, and as such, suggest that gaming disorder, as a diagnostic category, should be subject to ongoing investigation along with the accumulating evidence on the variety of related problems that people have-and how those problems are related to specific sociocultural factors and mental disorders, in order to avoid "medical ethnocentrism" (Glaser 1974) and "concept creeping" (Haslam 2016). As many problems seem to derive from gaming serving as an accessible and efficient means for compensating various adverse life scenarios, we hypothesize that titles such as WoW are not more "addictive" than other titles (by effect), 
but they are more prevalent in treatment-seekers' stories because they have large player bases and their multilayered designs are more likely to "match" with people's heterogenous difficulties and needs.

\section{Treatment-seekers}

Feeling of guilt or regret for having wasted time on gaming over experiences that would have had "real value"

\section{Esports amateurs}

Gaming is a high-investment meaningful routine

During our analysis, a new anthropological study on gaming in India was published (Snodgrass et al. 2021). The researchers studied 48 committed esports players, none of which, to our knowledge, had sought treatment for their gaming yet some self-identified as "addicted":

Gaming was the center of their social and aspirational lives, and so they demonstrated deep commitment to the activity. But they were also young men with future aspirations of having a good job and happy marriage. From that second point of view, they acknowledged the risks inherent in spending all their time in this way, which could lead them to judge as addictive the very experiences that lent their current lives purpose and meaning ... speaking in the language of addiction helped our respondents transition to their next householder stage of life by distancing them from their past

Snodgrass and colleagues, with reference to previous medical anthropology (see Dressler 2022), coined the term "cultural dissonance" to explain how self-diagnosed addiction serves as a means for the Indian esports players to make sense of the conflict between gaming and what the local culture expected from them. Namely, holding the belief that their gaming was addicted and hindered meeting the norms of the Indian society helped them to resolve the paradox of esports being valuable for them but not for the culture in which they lived. In earlier work, Snodgrass and colleagues $(2011 ; 2014)$ summarize:

problematic play emerges when gamers feel themselves better positioned in an online social world compared to an offline one and come to believe-either rightly, through a realistic assessment of less-than-desirable offline opportunities, or wrongly, through misplaced rationalization-that online life offers more fulfilling possibilities (2014, p. 496)

Based on the present analysis, we corroborate "cultural dissonance" as a recurring phenomenon. Many of our key findings tangle around the participants' value hierarchies, which for most treatment-seekers had undergone critical reconstruction from gamingcentered to culture-centered systems. Contrarily, our esports players-some of whom were significantly older than the treatment-seekers-had maintained gaming among their core life values and playing several hours daily was a meaningful part of their lives. We witness how gaming-related health problems are not simply in people but in the interactions that people have with specific environments in specific sociocultural contexts. As reiterated by Bosman (2017):

[Pathology] is the diminished capacity of the organism in that particular environment to conform to existing norms (i.e., to make life profitable for survival or pleasurable). 
In other words, pathology emerges when organism A in environment B is unable to conform to norm C. Because pathology is an emergent property of the relationship between the organism and its environment, a pathology or disorder cannot be assigned to a characteristic of the organism or the environment (pp. 49-50)

While our exploratory study is merely among the first steps toward an understanding of what gaming disorder might be, we are already beginning to see theoretical avenues and directions that could be taken to make sustainable progress in future research (Figure 1). Although individual biopsychosocial differences do likely contribute to the (lack of) problems that people have with gaming, research must also incorporate the material environment (home, school, videogame design, etc.) and culture (norms, society, stigmatization, etc.)-in the context of biographic, processual interactions (see Perales et al. 2020; Wakefield 1992). At the same time when the problems related to gaming seem to be associated with how some people feel (dis)positioned in culture and society, for others

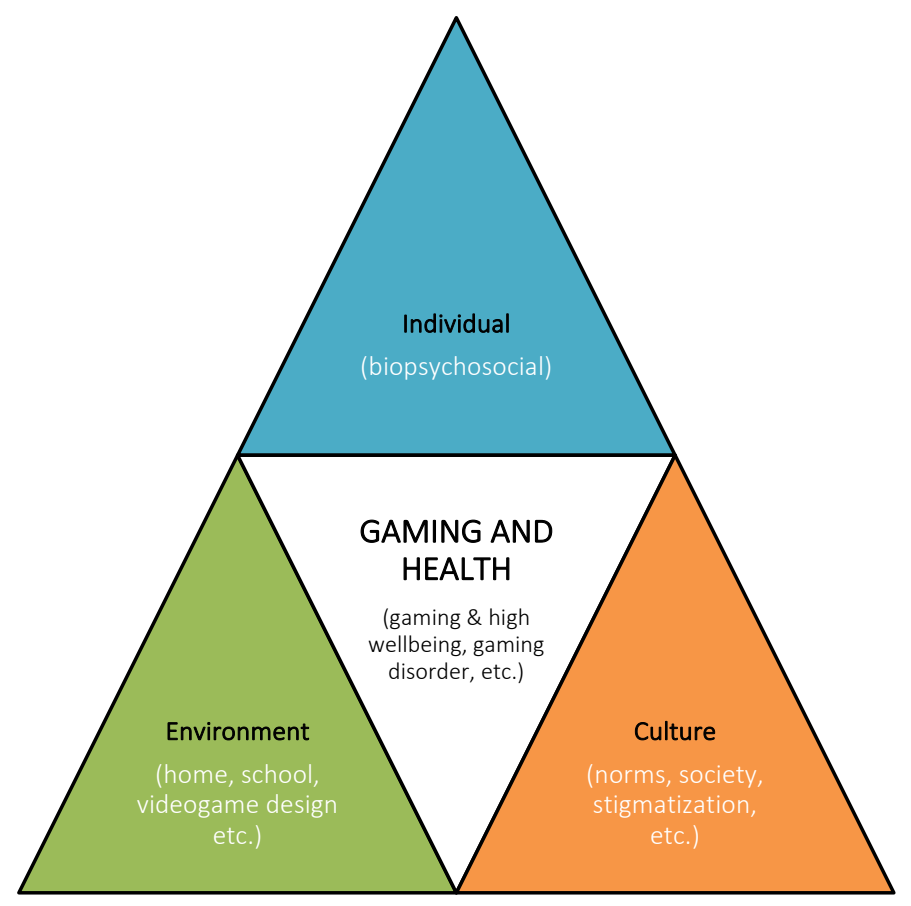

Figure 1. A framework for researching gaming and wellbeing, based on our findings and literature. More explicit attention should be given to the surrounding, material environments and cultural contexts. the problems appear to be strongly tied to preceding mental disorders and lacking self-regulation (note recent case studies in particular: Singh \& Raut 2022; Siste et al. 2020). This suggests that there could be numerous different types of health problems related to gaming, while many of them weakly related to each other (see Stepney 1996; McWilliams 2021). Addressing these problems collectively as a single "gaming disorder" will continue challenging both clinicians and researchers because the presumably wide spectrum of related problems-not all of which clinically significant-remain unknown, thus complicating measures as well as theory.

\section{Instruments, Limitations, and New Hypotheses}

Fourteen of our 16 participants also filled out three validated screening instruments for gaming-related disorders (Appendix 2). A fourth instrument was also filled: a single-item measure (THL1) developed by the Finnish National Institute of Health, which asks the respondent how often they have gaming-related problems ("never", "sometimes", "often", "almost always"). The most efficient tool for identifying treatment-seekers was THL1: three treatment-seekers self-reported to have problems "often" or "almost always" (zero esports players), while only two treatment seekers met the official GAS7 cutoff (zero esports players), one treatment-seeker met the official IGDT-10 cutoff (zero esports players), and zero treatment-seekers met the official GDT cutoff (zero esports players). 
Although our study was not designed to test validated screening instruments, these data contribute to the interpretation of epidemiological findings; namely, most people who experience gaming-related health problems and seek help for them do not seem to meet psychometrically validated criteria, but rather the best way to identify them seems to be their self-assessment of problems (THL1). In fact, during participant checking, one of the treatment-seekers told us that the scales did not measure their gaming problems due to poor verbal phrasings related to time. Based on our qualitative findings, we suggest that one key reason for the validated instruments to be inefficient for identifying treatment-seekers is that they clearly measure multiple "problem" (and non-problem) dimensions at once; moreover, as our participants were already seeking treatment, official diagnostic criteria such as increasing interest in gaming simply did not apply. On the other hand, none of our esports players met any of the cutoffs either, which is comforting regarding the fears of false positive diagnoses.

As we were carrying out our analysis, the WHO added a developmental outline of their gaming disorder diagnosis, which starts as follows: "Gaming Disorder appears to be most prevalent among adolescent and young adult males aged 12 to 20 years" (https://icd.who.int/en). Because all our participants were older than 20 years and many of them past 30, our sample does not represent the current description of the default gaming disorder. To this point, we add that the adult experiences analyzed in the present study-as represented by subordinate themes reflecting intensive life and work events with subjective value reconstructionscannot, by definition, have much to do with the experiences of children below 15 years. It remains for future research to investigate if and how the dominant diagnosis of gaming disorder (in children) is related to our findings.

Finally, in Bayesian spirit, we update our beliefs and set new hypotheses. First, while our trust in neglected needs/responsibilities and loss of control as the core aspects of gaming-related health problems remains strong $(\mathrm{QH} 1)$, we further hypothesize that-in the current era of cultural and technological progress-such problems (as defined by clinical treatment-seeking) are a unique outcome of having faced abuse, discrimination, or other forms of mistreatment in childhood and/or adolescence development, which has made gaming meaningful in a protective, coping-type way. This restricted meaningfulness could contribute to "social thinning" (McCrory et al. 2022), which hinders the development of resilience-providing networks. Treatment providers could ask seekers to fill out related questionnaires in order to help generating large-scale data for testing this hypothesis. If corroborated, the hypothesis could be utilized for distinguishing help-needing players from intensively gaming healthy individuals, and most importantly, for helping youthwork practitioners in providing support for those adolescents who may not have had optimal environments for their critical early years of development.

Second, compared to our priors, we now believe "self-development" to be less commonly a central meaning component for both treatment-seekers and esports players, as even some of the latter explicitly expressed their gaming to be meaningful for other reasons ( $\mathrm{QH} 2)$. Likewise, individuals in both groups expressed significant life conflicts-but only the latter had been able to efficiently resolve them. As a new hypothesis, we propose that gaming-related treatment-seeking in adulthood is significantly connected to a reconstruction of personal 
values. This may manifest, for instance, as the loss of meaning in previously valued gaming, the emergence of new values in one's life situation, or unexpected events that significantly affect the individual. Again, multicultural clinical adult samples are needed to properly test the hypothesis (for further recent evidence, see Karhulahti et al. 2022b). If corroborated, the hypothesis would lead to a refined understanding of how seeking treatment for one's gaming can be explained by the complex interplay of cultural, environmental, and individual factors (e.g., Kleinman 1977). Ultimately, a better understanding of the phenomenon will improve the development of appropriate support systems, which may not necessarily be best driven by gaming-specific psychiatric diagnoses.

\section{Conclusions}

Although gaming-related health problems have been studied since the 1980s, a lacking understanding regarding what such problems are still defines the field because no in-depth phenomenological studies have been carried out with actual treatment-seekers. To start building a basis for understanding what gaming-related health problems are, our comparative study between treatment-seekers and intensively gaming esports players suggests "gaming disorder" experiences to essentially derive from gaming interfering with what one wants to be, do, and have, whereas for people who play several daily hours without problems gaming experiences represent what they want to be, do, and have. We also found several critical differences within the experiences of both groups, which implies that the diagnostic criteria of "gaming disorder" - which are now primarily designed to describe cases of children and young adolescents-might not be able to capture adult treatment-seekers. To pursue a better scientific understanding of the issue, qualitative studies should be carried out in multiple age groups, countries, and cultural contexts. In a distant future where dozens of such studies have been carried out, meta-analyses will hopefully be able to identify universal thematic patterns, if they exist. To our knowledge, this was the first registered report using phenomenological qualitative methods. We encourage researchers to further explore the method-specific utility of registered reports, which can help us solve "gaming disorder" collectively, as a cumulative scientific enterprise.

\section{Data availability}

The transcripts have been moved to the Finnish Social Science Data Archive for scientific reuse. Because this is a longitudinal study, according to regional data policy, VMK must confirm all requests for data access until the longitudinal project has ended and all pseudonym identifiers have been permanently erased (at this point, the data become directly accessible to all educational and research purposes). All requests motivated by secondary analysis or deeper understanding of the findings will be accepted, and to ensure fair sharing, Recommender can be contacted if any request would be rejected on unfair grounds.

\section{Code availability}


The idiographic coding process was documented by each interviewee (case summaries with participant-specific themes and codes). Due to the privacy concerns related to the qualitative nature of the data, some parts of the original coding documents have been removed to protect participants.

\section{References}

Bean, A. M., Nielsen, R. K., Van Rooij, A. J., \& Ferguson, C. J., (2017) Video game addiction: the push to pathologize video games. Prof Psychol-Res Pr, 48, 10.1037/pro0000150 Billieux, J., Flayelle, M., Rumpf, H. J., \& Stein, D. J. (2019) High involvement versus pathological involvement in video games: a crucial distinction for ensuring the validity and utility of gaming disorder. Curr Addict Rep, 6, 323-330

Bishop, L. \& Kuula-Luumi, A. (2017) Revisiting qualitative data reuse: a decade on. Sage Open, 7, 2158244016685136

Blaszczynski, A., \& Nower, L. (2002). A pathways model of problem and pathological gambling. Addiction, 97(5), 487-499.

Boonen, C. S., Christiansen, M. V., Ilsøe, A. W., (2018) Staunstrup, M. M., \& Nielsen, R. K. Contextualizing pathological gaming - a proof-of-concept study. In Proc DiGRA'18. Bosman, A. M. (2017). Disorders are reduced normativity emerging from the relationship between organisms and their environment. In Parental responsibility in the context of neuroscience and genetics (pp. 35-54). Springer, Cham.

Brand, M., Wegmann, E., Stark, R., Müller, A., Wölfling, K., Robbins, T. W., \& Potenza, M. N. (2019). The Interaction of Person-Affect-Cognition-Execution (I-PACE) model for addictive behaviors: Update, generalization to addictive behaviors beyond internet-use disorders, and specification of the process character of addictive behaviors. Neuroscience \& Biobehavioral Reviews, 104, 1-10.

Branney, P., Reid, K., Frost, N., Coan, S., Mathieson, A., \& Woolhouse, M. (2019). A contextconsent meta-framework for designing open (qualitative) data studies. Qualitative Research in Psychology.

Braun, V., \& Clarke, V. (2021). To saturate or not to saturate? Questioning data saturation as a useful concept for thematic analysis and sample-size rationales. Qualitative research in sport, exercise and health, 13(2), 201-216.

Brevers, D., King, D. L., \& Billieux, J. (2020) Delineating adaptive esports involvement from maladaptive gaming: a self-regulation perspective. Current Opinion in Psychology, 36, 141146

Carbonell, X. (2017) From Pong to Pokemon go, catching the essence of the Internet gaming disorder diagnosis. Journal of Behavioral Addictions, 6, 124-127

Castro-Calvo, J., King, D. L., Stein, D. J., Brand, M., Carmi, L., Chamberlain, S. R., et al. (2021)

Expert appraisal of criteria for assessing gaming disorder: An international Delphi study.

Addiction, https://doi.org/10.1111/add.15411

Ceranoglu, T. A. (2010) Video games in psychotherapy. Review of General Psychology, 14, 141-146

Charlton, J. P. \& Danforth, I. D. (2007) Distinguishing addiction and high engagement in the context of online game playing. Comput hum behav, 23, 1531-1548

Clark, L., Billieux, J., \& Ledgerwood, D. M. (2022). Introduction to the Special Issue "20 years of the Pathways Model: understanding disordered gambling and other behavioural addictions". International Gambling Studies, 22(2), 183-187. 
Colder Carras, M., et al. (2018) Gamers' insights into the phenomenology of normal gaming and game "addiction": a mixed methods study. Comput hum behav, 79, 238-246

Carras, M. C. \& Kardefelt-Winther, D. (2018). When addiction symptoms and life problems diverge: A latent class analysis of problematic gaming in a representative multinational sample of European adolescents. European Child \& Adolescent Psychiatry, 27 (4), 513-525. Chambers, R. A., Taylor, J. R., \& Potenza, M. N. (2003). Developmental neurocircuitry of motivation in adolescence: a critical period of addiction vulnerability. American journal of psychiatry, 160(6), 1041-1052.

Domahidi, E., \& Quandt, T. (2015) "And all of a sudden my life was gone...": A biographical analysis of highly engaged adult gamers. new media \& society, 17, 1154-1169

Dressler, W. (2022) Culture and the stress process. In M. Singer \& P. Erickson (Eds.), A Companion to Medical Anthropology ( $2^{\text {nd }}$ Ed.). Wiley-Blackwell.

Ferguson, C. J., Coulson, M., \& Barnett, J. (2011) A meta-analysis of pathological gaming prevalence and comorbidity with mental health, academic and social problems. Journal of psychiatric research, 45, 1573-1578

Ferguson, C. J. \& Colwell, J. (2020) Lack of consensus among scholars on the issue of video game "addiction". Psychol Pop M, 9, 359-366

Foddy, B. \& Savulescu, J. (2010), "A Liberal Account of Addiction," Philosophy, Psychiatry \& Psychology, 17 (1): 1-22.

Fried, E. I., van Borkulo, C. D., Cramer, A. O., Boschloo, L., Schoevers, R. A., \& Borsboom, D. (2017). Mental Disorders as Networks of Problems: A Review of Recent Insights. Social Psychiatry and Psychiatric Epidemiology, 52 (1). https://doi.org/10.1007/s00127-016-1319-z García-Lanzo, S., \& Chamarro, A. (2018). Basic psychological needs, passion and motivations in amateur and semi-professional eSports players. Aloma: revista de psicologia, ciències de l'educació i de l'esport Blanquerna, 36(2), 59-68.

Glaser, F. B. (1974). Medical ethnocentrism and the treatment of addiction. International Journal of Offender Therapy and Comparative Criminology, 18(1), 13-27.

Granic, I., Lobel, A., \& Engels, R. C. (2014) The benefits of playing video games. Am psychol, 69, 66-78

Griffiths, M. D., et al. (2016) Working towards an international consensus on criteria for assessing Internet gaming disorder: a critical commentary on Petry et al. 2014. Addiction, 111, 167-175

Götzenbrucker, G., \& Köhl, M. (2009) Ten years later. Eludamos. Journal for Computer Game Culture, 3, 309-324

Hacking, I. (2004). Between Michel Foucault and Erving Goffman: Between Discourse in the Abstract and Face-to-face Interaction. Economy and Society, 33 (3).

Haslam, N. (2016). Concept creep: Psychology's expanding concepts of harm and pathology. Psychological Inquiry, 27(1), 1-17.

Haven, T.L., Errington, T.M., Gleditsch, K.S., van Grootel, L., Jacobs, A.M., Kern, F.G., et al. (2020) Preregistering qualitative research: a delphi study. Int J Qual Met, 19, 1609406920976417

Haven, T., \& Van Grootel, D. L. (2019) Preregistering qualitative research. Account Res, 26, 229-244

Hennink, M. M., Kaiser, B. N., \& Marconi, V. C. (2017) Code Saturation Versus Meaning Saturation: How Many Interviews Are Enough? Qualitative Health Research, 27, 591- 608 
Himmelstein, D., Liu, Y., \& Shapiro, J. L. (2021) An exploration of mental skills among competitive league of legend players. Research Anthology on Rehabilitation Practices and Therapy, 1607-1629

Jacobs, D. F. (1986). A general theory of addictions: A new theoretical model. Journal of gambling behavior, 2(1), 15-31.

Jeong, H., Yim, H. W., Lee, S. Y., Lee, H. K., Potenza, M. N., Kwon, J. H., et al. (2018)

Discordance between self-report and clinical diagnosis of Internet gaming disorder in adolescents. Scientific reports, 8, 1-8

Jeong, E. J., Ferguson, C. J., \& Lee, S. J. (2019) Pathological gaming in young adolescents: A longitudinal study focused on academic stress and self-control in South Korea. Journal of youth and adolescence, 48, 2333-2342

Johannes N., Vuorre M. \& Przybylski A. K. (2021) Video game play is positively correlated with well-being. R. Soc. open sci. 8202049

Kardefelt-Winther, D. (2017) Conceptualizing internet use disorders: addiction or coping process? Psychiat Clin Neuros, 71, 459-466

Karhulahti, V. (2020) Esport Play: Anticipation, Attachment, and Addiction in Psycholudic Development. Bloomsbury Publishing: New York

Karhulahti, V. (2022) Three Reasons for Qualitative Psychologists to Share Human Data. Preprint, British Journal of Social Psychology. DOI: 10.1111/bjso

Karhulahti, V., Martončik, M., \& Adamkovic, M. (2021). Measuring Internet Gaming Disorder and Gaming Disorder: Qualitative Content Validity Analysis of Validated Scales. PsyArXiv. https://doi.org/10.31234/osf.io/dgtmq Karhulahti, V., Nerg, H., Laitinen, T., Päivinen, A., \& Chen, Y. (2022a). Eight Hypotheses on Technology Use and Mental Wellbeing: A Bicultural Phenomenological Study of Gaming during the COVID-19 Pandemic. Current Psychology. DOI: 10.1007/s12144-022-03586-x Karhulahti, V. M., Nuutinen, S., \& Lukka, L. (2022). Why do adults seek treatment for gaming (disorder)? A qualitative study. PsyArXiv. https://psyarxiv.com/e7a6k/ Karlsen, F. (2013) A world of excesses: Online games and excessive playing. Routledge: Abingdon

Kearney, M. H., \& O'Sullivan, J. (2003). Identity shifts as turning points in health behavior change. Western Journal of Nursing Research, 25(2), 134-152.

King, D. L., Chamberlain, S. R., Carragher, N., Billieux, J., Stein, D., Mueller, K., et al. (2020) Screening and assessment tools for gaming disorder: a comprehensive systematic review. Clin Psych Rev, 77, 101831

King, D. L., \& Delfabbro, P. (2009). Understanding and assisting excessive players of video games: A community psychology perspective. Australian Community Psychologist, 2, 62-74 Kleinman, A. M. (1977). Depression, somatization and the "new cross-cultural psychiatry". Social Science \& Medicine (1967), 11(1), 3-9.

Kleinman, A. (1988). Rethinking psychiatry: From cultural category to personal experience. The Free Press.

Kneer, J., Rieger, D., Ivory, J. D., \& Ferguson, C. (2014) Awareness of risk factors for digital game addiction: interviewing players and counselors. Int J Ment Health Ad, 12, 585-599 Ko, C. H., Lin, H. C., Lin, P. C., \& Yen, J. Y. (2020). Validity, functional impairment and complications related to Internet gaming disorder in the DSM-5 and gaming disorder in the ICD-11. Australian \& New Zealand Journal of Psychiatry, 54(7), 707-718.

Koski-Jännes, A. (2002). Social and personal identity projects in the recovery from addictive behaviours. Addiction Research and Theory, 10(2), 183-202. 
Kou, Y., \& Gui, X. (2020) Emotion regulation in esports gaming: a qualitative study of league of legends. CSCW2, 4, 1-25

Kowert, R., Domahidi, E., \& Quandt, T. (2014) The relationship between online video game involvement and gaming-related friendships among emotionally sensitive individuals.

Cyberpsychology, Behavior, and Social Networking, 17, 447-453

Kowert, R., Vogelgesang, J., Festl, R., \& Quandt, T. (2015) Psychosocial causes and consequences of online video game play. Comp Hum Behav, 45, 51-58

Larkin, M., \& Thompson, A. R. (2012) Interpretative phenomenological analysis in mental health and psychotherapy research In. Qualitative Research Methods in Mental Health and Psychotherapy (Thompson, A. \& Harper, D. ed.) 101-116. John Wiley \& Sons: San Francisco. Maslow, A. H. (1943). A theory of human motivation. Psychological review, 50(4), 370. Matthews, S., Dwyer, R., \& Snoek, A. (2017). Stigma and Self-Stigma in Addiction. Journal of Bioethical Inquiry, 1-12.

McCrory, E., Foulkes, L., \& Viding, E. (2022). Social thinning and stress generation after childhood maltreatment: a neurocognitive social transactional model of psychiatric vulnerability. The Lancet Psychiatry.

McWilliams, N. (2021). Diagnosis and Its Discontents: Reflections on Our Current Dilemma. Psychoanalytic Inquiry, 41(8), 565-579.

Mitchell, S. D. (2009). Unsimple truths. University of Chicago Press.

Nielsen, R. \& Karhulahti, V. (2017) The problematic coexistence of internet gaming disorder and esports. in Proc FDC'12. ACM

Noë, A. (2009). Out of our heads: Why you are not your brain, and other lessons from the biology of consciousness. Macmillan.

Olthof, M., Hasselman, F., Maatman, F. O., Bosman, A., \& Lichtwarck-Aschoff, A. (2022). Complexity theory of psychopathology. Journal of Psychopathology and Clinical Science. Partanen, J. (1996). Why alcohol and drug research remains a marginal activity. Addiction, 91(2), 225-226.

Pellegrini, A. (2009) The Role of Play in Human Development. Oxford University Press Perales, J. C., King, D. L., Navas, J. F., Schimmenti, A., Sescousse, G., Starcevic, V., ... \& Billieux, J. (2020). Learning to lose control: A process-based account of behavioral addiction. Neuroscience \& Biobehavioral Reviews, 108, 771-780.

Piaget, J. (2013) Play, Dreams, and Imitation in Childhood (trans. Gattegno, C. \& Hodgson, F.). Routledge: Abingdon Przybylski, A. (2014) Electronic gaming and psychosocial adjustment. Pediatrics, 134, e716e722

Quandt, T., Grueninger, H., \& Wimmer, J. (2009) The gray haired gaming generation: Findings from an explorative interview study on older computer gamers. Games and Culture, 4, 27-46

Reiss, S. (2012). Intrinsic and extrinsic motivation. Teaching of psychology, 39(2), 152-156.

Rodriguez, L., \& Smith, J. A. (2014). 'Finding your own place': an interpretative phenomenological analysis of young men's experience of early recovery from addiction. International Journal of Mental Health and Addiction, 12(4), 477-490.

Ryan, R. \& Deci, E. (2017) Self-determination Theory: Basic Psychological Needs in Motivation, Development, and Wellness. Guilford Publications.

Satchell, L.P., Fido, D., Harper, C.A. et al. Development of an Offline-Friend Addiction Questionnaire (O-FAQ): Are most people really social addicts?. Behav Res 53, 1097-1106 (2021). https://doi.org/10.3758/s13428-020-01462-9 
Saunders, B., Sim, J., Kingstone, T., Baker, S., Waterfield, J., Bartlam, B., et al. (2018)

Saturation in qualitative research: exploring its conceptualization and operationalization.

Quality and Quantity, 52, 1893-1907

Schimmenti, A., Passanisi, A., Gervasi, A. M., Manzella, S., \& Famà, F. I. (2014). Insecure attachment attitudes in the onset of problematic Internet use among late adolescents. Child Psychiatry \& Human Development, 45(5), 588-595.

Shi, J., Renwick, R., Turner, N. E., \& Kirsh, B. (2019). Understanding the lives of problem gamers: The meaning, purpose, and influences of video gaming. Computers in Human Behavior, 97, 291-303.

Shinebourne, P., \& Smith, J. A. (2009). Alcohol and the self: An interpretative phenomenological analysis of the experience of addiction and its impact on the sense of self and identity. Addiction Research \& Theory, 17(2), 152-167.

Shinebourne, P., \& A Smith, J. (2011a). 'It is just habitual': an interpretative phenomenological analysis of the experience of long-term recovery from addiction. International Journal of Mental Health and Addiction, 9(3), 282-295.

Shinebourne, P., \& Smith, J. A. (2011b). Images of addiction and recovery: An interpretative phenomenological analysis of the experience of addiction and recovery as expressed in visual images. Drugs: education, prevention and policy, 18(5), 313-322.

Singer, M. (2012). Anthropology and addiction: an historical review. Addiction, 107(10), 1747-1755.

Singh, S., \& Raut, N. (2022). Internet Gaming Disorder: A Real Disorder or a Way of Coping?

A Case Series. Journal of Indian Association for Child and Adolescent Mental Health, 09731342221096470.

Siste, K., Hanafi, E., Jamtani, D., Murtani, B. J., Beatrice, E., Christian, H., ... \& Ismail, R. I. (2020). Gaming disorder and parenting style: A case series. Addictive Disorders \& Their Treatment, 19(3), 185-190.

Siutila, M., \& Karhulahti, V. (2021) Continuous play: leisure engagement in competitive fighting games and taekwondo. Ann Leisure Res. 10.1080/11745398.2020.1865173 Skog, O. J. (2000). Addicts' choice. Addiction, 95(9), 1309-1314.

Smith, J. A. (1996) Beyond the divide between cognition and discourse: Using interpretative phenomenological analysis in health psychology. Psychology and health, 11, 261-271

Smith, J. A., Jarman, M., \& Osborn, M. (1999) Doing interpretative phenomenological analysis. Qualitative Health Psychology: Theories and Methods (eds. Murray, M. \& Chamberlain, K.) 218-240. Sage: London

Smith, J. A., \& Osborne, P. (2007) Interpretative phenomenological analysis. In Qualitative Psychology: A Practical Guide to Research Methods (Smith, J. ed.) 25-52. Sage: London Smith, J., Larkin, M., \& Flowers, P. (2009). Interpretative Phenomenological Analysis Theory, Method and Research. Sage.

Snodgrass, J. G., Lacy, M. G., Dengah, H. J., \& Fagan, J. (2011). Cultural consonance and mental wellness in the world of warcraft: online games as cognitive technologies of 'absorption-immersion'. Cognitive Technology, 16 (1), 11-23.

Snodgrass, J. G., Dengah, H. F., \& Lacy, M. G. (2014). "I swear to God, I only want people here who are losers!" Cultural dissonance and the (problematic) allure of Azeroth. Medical Anthropology Quarterly, 28(4), 480-501.

Snodgrass, J., Bagwell, A., Patry, J. M., Dengah II, H. F., Smarr-Foster, C., Van Oostenburg, M., et al. (2018) The partial truths of compensatory and poor-get-poorer internet use 
theories: More highly involved videogame players experience greater psychosocial benefits. Comp Hum Behav, 78, 10-25

Snodgrass, J. G., Dengah, H. F., Polzer, E., \& Else, R. (2019) Intensive online videogame involvement: A new global idiom of wellness and distress. Transcultural Psychiatry, 56, 748774

Snodgrass, J. G., Dengah, H. F., Upadhyay, C., Else, R. J., \& Polzer, E. (2021). Indian Gaming Zones as Oppositional Subculture: A Norm Incongruity "Cultural Dissonance" Approach to Internet Gaming Pleasure and Distress. Current Anthropology, 62(6).

Sripada, C. (2022). Impaired control in addiction involves cognitive distortions and unreliable self-control, not compulsive desires and overwhelmed self-control. Behavioural Brain Research, 418, 113639.

Starcevic, V., Choi, T. Y., Kim, T. H., Yoo, S. K., Bae, S., Choi, B. S., et al. (2020) Internet gaming disorder and gaming disorder in the context of seeking and not seeking treatment for video-gaming. Journal of psychiatric research, 129, 31-39

Stavropoulos, V., Gomez, R., \& Motti-Stefanidi, F. (2019). Internet Gaming Disorder: A Pathway Towards Assessment Consensus. Frontiers in psychology, 10, 1822.

Stepney, R. (1996). The concept of addiction: its use and abuse in the media and science. Human Psychopharmacology: Clinical and Experimental, 11(1), 15-20.

Syed, M., \& Nelson, S. C. (2015) Guidelines for establishing reliability when coding narrative data. Emerging Adulthood, 3, 375-387

Vahlo, J., Karhulahti, V. M., \& Koponen, A. (2018) Tasavallan core-gamer: videopelaamisen piirteet Suomessa, Kanadassa ja Japanissa. Pelitutkimuksen vuosikirja, 35-59

Van Leeuwen, L., \& Westwood, D. (2008) Adult play, psychology and design. Digit Creat, 19, 153-161

Van Manen, M., Higgins, I., Van der Reit, P. (2016) A conversation with Max van Manen on phenomenology in its original sense. Nurs. Health Sci, 18, 4-7

Van Rooij, A. J., Ferguson, C. J., Colder Carras, M., Kardefelt-Winther, D., Shi, J., Aarseth, E., ... Przybylski, A. K. (2018). A weak scientific basis for gaming disorder: Let us err on the side of caution. Journal of Behavioral Addictions, 1-9. https://doi.org/10.1556/2006.7.2018.19 Van Rooij, A. J., Ferguson, C. J., Van de Mheen, D., \& Schoenmakers, T. M. (2017) Time to abandon internet addiction? predicting problematic internet, game, and social media use from psychosocial well-being and application use. Clin Neuropsychiatry, 14, 113-121 Wakefield, J. C. (1992). The concept of mental disorder: on the boundary between biological facts and social values. American psychologist, 47(3), 373.

Wakefield, J. C. (2020). Addiction from the harmful dysfunction perspective: How there can be a mental disorder in a normal brain. Behavioural Brain Research, 389, 112665.

West, R., \& Brown, J. (2013). Theory of addiction. Wiley Blackwell.

WHO (The World Health Organization) (2021) International Statistical Classification of Diseases and Related Health Problems (ICD). Official Website.

https://www.who.int/standards/classifications/classification-of-diseases

\section{Acknowledgements}

The work was supported by the Finnish Work Environment Fund (200349), Academy of Finland (312397), and the European Research Council (ERC) under the European Union's Horizon Europe research and innovation programme (101042052). 
Warm thanks go to all participants who made this study possibly by sacrificing significant amounts of their time and agreed to share their answers for reuse. We also thank all collaborating institutions and individuals that enabled us to find participants. Credit for helping with proofreading and transcription: Valtteri Kauraoja, Soli Perttu, Tanja Laitinen, Venla Korhonen.

\section{Author contributions}

Conceptualization: VMK. Data curation: VMK, MS. Formal analysis: VMK, MS. Funding Acquisition: VMK, JV, RK. Investigation: VMK, MS, JV, RK. Methodology: VMK. Project administration: VMK. Resources: VMK, RK. Software: VMK, MS. Supervision: VMK.

Validation: VMK, JV, RK. Visualization: MS. Writing original draft: VMK. Writing review \& editing: VMK, MS, JV, RK.

\section{Competing interests}

Veli-Matti Karhulahti is one of the PCI Registered Reports recommenders. JV is a CSO in Kinrate Analytics, which provides personalized game recommendations. 
Methodological details (Group 1 and 2) according to

Tong A, Sainsbury P, Craig J. Consolidated criteria for reporting qualitative research (COREQ): a 32-item checklist for interviews and focus groups. International journal for quality in health care. 2007 Dec 1;19(6):349-57.

\section{Interviewer/facilitator}

MS and VMK conducted all interviews.

\section{Credentials}

MS has an MA (media studies) and VMK has a PhD (title of a docent in gaming research).

\section{Occupation}

VMK is a senior researcher in University of Jyväskylä and MS is a PhD candidate in University of Turku.

\section{Gender}

MS identifies as a woman and VMK identifies as a man.

\section{Experience and training}

Both authors are trained researchers with previous interviewing experience.

\section{Relationship established}

Authors have not communicated with the interviewees before.

\section{Participant knowledge of the interviewer}

The purpose of the research was shared with the interviewees before the interview, and the interviewees were invited to ask questions related to the study before the interview. The authors did not systematically disclose their own histories, but when asked, they shared those openly.

\section{Interviewer characteristics}

MS and VMK are familiar with videogames, including esports. MS and VMK believe that gaming can be related to health problems and benefits in complex ways that are not yet well modeled or explained theoretically. The presented QH1-3 list selected literature that directly influence this specific study. Any related questions posed by the interviewees to the interviewers were answered transparently (outside recording).

\section{Methodological orientation and theory}

Interpretive phenomenological analysis, with an added phenomenological manual that we used for systematically documenting the development of structural connections between cases and thematic iteration.

\section{Sampling}

Purposive sampling.

\section{Method of approach}

Open call distributed via collaborators and special institutions.

\section{Sample size}

See Table 1.

\section{Non-participation}

$\mathrm{N} / \mathrm{A}$

\section{Setting of data collection}


Remotely from home office. A pilot interview, with MS and VMK both present, was carried out with an esports-playing individual using the POP before starting data collection.

15. Presence of non-participants

N/A

\section{Description of sample}

See Table 1.

17. Interview guide

Phenomenology of Play (POP).

18. Repeat interviews

Follow-up in 12 months (not reported in this study).

19. Audio/visual recording

Audio recording.

20. Field notes

Personal notes were taken and discussed after each interview.

21. Duration

Average interview length was expected to be 120 minutes and ended up being 112 minutes.

22. Data saturation

Not used.

23. Transcripts returned

Transcripts were shared with participants for verification; the participants could choose parts of the transcripts not to be shared openly for later scientific use (but we did use all parts in our analysis).

24. Number of data coders

Lead coders (MS and JV) and assistant coders (RK).

25. Description of the coding tree

N/A

\section{Derivation of themes}

IPA-driven thematization with a phenomenological manual for documenting progress.

\section{Software}

Zoom for interviews and Atlas.ti for coding.

\section{Participant checking}

Member checking was further applied by providing, during transcript checks, also our tentative interpretations in written format, and the participants were given the opportunity to comment on them. We took the commentary into consideration as reflective follow-up data. In the follow-up interview, the previous findings and our interpretations are discussed verbally and the interviewees are asked to comment.

29. Quotations presented

Cited with permission, idiographic presentation.

30. Data and findings consistent

Two coders/interpreters for each case, and an external validation by third and fourth members.

31. Clarity of major themes

N/A

32. Clarity of minor themes

N/A 
Two participants did not fill in the screening survey. All screening tools used with permission. The survey is identical to the one used in a pilot for our other registered report (Karhulahti et al. 2022).

\begin{tabular}{|l|l|l|l|l|l|l|l|l|l|}
\hline & GAS7 & IGDT10 & GDT & THL1 & BDI & GAD7 & GH & BBDS & WAI \\
\hline Aaron & $3 / 7$ & $5 / 9$ & $2 / 4$ & $4 / 4$ & $12 / 18$ & $12 / 21$ & $3 / 5$ & $0 / 3$ & $4 / 10$ \\
\hline Bruno & $5 / 7$ & $2 / 9$ & $3 / 4$ & $2 / 4$ & $8 / 18$ & $14 / 21$ & $3 / 5$ & $0 / 3$ & $7 / 10$ \\
\hline Caius & $0 / 7$ & $0 / 9$ & $0 / 4$ & $1 / 4$ & $1 / 18$ & $0 / 21$ & $3 / 5$ & $0 / 3$ & $8 / 10$ \\
\hline Dan & $2 / 7$ & $0 / 9$ & $0 / 4$ & $2 / 4$ & $1 / 18$ & $1 / 21$ & $3 / 5$ & $0 / 3$ & $7 / 10$ \\
\hline Eetu & $4 / 7$ & $0 / 9$ & $0 / 4$ & $4 / 4$ & $9 / 18$ & $5 / 21$ & $3 / 5$ & $0 / 3$ & $6 / 10$ \\
\hline Fredrika & $3 / 7$ & $4 / 9$ & $3 / 4$ & $3 / 4$ & $0 / 18$ & $5 / 21$ & $4 / 5$ & $0 / 3$ & $7 / 10$ \\
\hline Gabriel & $1 / 7$ & $1 / 9$ & $2 / 4$ & $2 / 4$ & $0 / 18$ & $2 / 21$ & $4 / 5$ & $0 / 3$ & $9 / 10$ \\
\hline Heikki & $2 / 7$ & $1 / 9$ & $0 / 4$ & $1 / 4$ & $0 / 18$ & $2 / 21$ & $3 / 5$ & $0 / 3$ & $6 / 10$ \\
\hline Ida & $2 / 7$ & $1 / 9$ & $0 / 4$ & $2 / 4$ & $5 / 18$ & $3 / 21$ & $4 / 5$ & $0 / 3$ & $8 / 10$ \\
\hline Jaakko & & & & & & & & & \\
\hline Kalle & $1 / 7$ & $0 / 9$ & $0 / 4$ & $1 / 4$ & $1 / 18$ & $2 / 21$ & $3 / 5$ & $1 / 3$ & $8 / 10$ \\
\hline Lauri & $1 / 7$ & $1 / 9$ & $0 / 4$ & $1 / 4$ & $0 / 18$ & $0 / 21$ & $4 / 5$ & $0 / 3$ & $10 / 10$ \\
\hline Mikko & $3 / 7$ & $2 / 9$ & $0 / 4$ & $1 / 4$ & $4 / 18$ & $5 / 21$ & $3 / 5$ & $0 / 3$ & $8 / 10$ \\
\hline Niina & $3 / 7$ & $1 / 9$ & $0 / 4$ & $1 / 4$ & $9 / 18$ & $19 / 21$ & $3 / 5$ & $0 / 3$ & $10 / 10$ \\
\hline Oona & $0 / 7$ & $0 / 9$ & $0 / 4$ & $1 / 4$ & $0 / 18$ & $0 / 21$ & $5 / 5$ & $0 / 3$ & $9 / 10$ \\
\hline Pasi & & & & & & & & & \\
\hline
\end{tabular}

Gaming problems:

GAS7 (Lemmens et al. 2009)

- $\quad$ Based on pathological gambling in the DSM-IV. Scale 0-7, cutoff 4/7. IGDT10 (Király et al. 2017)

- Based on 'internet gaming disorder' in the third section of the DSM5. Scale 0-9, cutoff 5/9.

GDT (Pontes et al. 2019)

- Based on 'gaming disorder' in the ICD-11. Scale 0-4, cutoff 4/4.

THL1 (Salonen \& Raisamo 2015)

- One-item gaming problems self-assessment without disclosed basis. Scale 1-4.

The Finnish translations have all been found valid in previous research (Männikkö et al. 2015; Männikkö et al. 2019; Karhulahti \& Koskimaa 2019), except for GDT.

Other health:

BBDS (Gebauer et al. 2010)

- Gambling, Finnish version (Salonen \& Raisamo 2015). Scale 0-3, cutoff 1/3. 
BDI6 (Beck et al. 1996)

- Depression, Finnish version (Aalto et al. 2012). Scale 0-18.

GAD7 (Spitzer et al. 2006)

- Anxiety, Finnish version (Kujanpää et al. 2012). Scale 0-21.

GH PROMIS (Hays et al. 2009)

- General health, Finnish version (THL 2021). We report Global-1 (1-5) in the Table. WAI (Ilmarinen 2007)

- Work ability index, short Finnish version (von Bonsdorff et al. 2012). Scale 0-10.

\section{Sources}

Aalto, A. M., Elovainio, M., Kivimäki, M., Uutela, A., \& Pirkola, S. (2012). The Beck Depression Inventory and General Health Questionnaire as measures of depression in the general population: a validation study using the Composite International Diagnostic Interview as the gold standard. Psychiatry research, 197(1-2), 163-171.

Beck, A. T., Steer, R. A., \& Brown, G. (1996). Beck depression inventory-II. BDI-II manual. San Antonio, TX: The Psychological Corporation.

Gebauer L, LaBrie R, Shaffer HJ. Optimizing DSM-IV-TR Classification accuracy: A brief Biosocial Screen for Detecting Current gambling Disorders Among Gamblers in the General Household Population. The Canadian Journal of Psychiatry 2010; 55(2): 82-90. Hays, R. D., Bjorner, J. B., Revicki, D. A., Spritzer, K. L., \& Cella, D. (2009). Development of physical and mental health summary scores from the patient-reported outcomes measurement information system (PROMIS) global items. Quality of Life Research, 18(7), 873-880.

IImarinen, J. (2007). The work ability index (WAI). Occupational medicine, 57(2), 160. Karhulahti, V. M., \& Koskimaa, R. (2019). On the Prevalence of Addicted or Problematic Gaming in Finland. Addictive behaviors reports, 10, 100225. Karhulahti, V. M., Vahlo, J., Martončik, M., Munukka, M., Koskimaa, R., \& von Bonsdorff, M. (2022). Ontological Diversity in Gaming Disorder Measurement: A Nationally Representative Registered Report. Addiction Research \& Theory.

https://doi.org/10.1080/16066359.2022.2115033

Kinnunen, U. \& Mauno, S. (2009) (eds.) Irtiottoja työstä. Työkuormituksesta palautumisen psykologia. Tampere: Yliopistopaino.

Király, O., Sleczka, P., Pontes, H. M., Urbán, R., Griffiths, M. D., \& Demetrovics, Z. (2017). Validation of the ten-item Internet Gaming Disorder Test (IGDT-10) and evaluation of the nine DSM-5 Internet Gaming Disorder criteria. Addictive behaviors, 64, 253-260. Kujanpää, T., Ylisaukko-Oja, T., Jokelainen, J., Hirsikangas, S., Kanste, O., Kyngäs, H., \& Timonen, M. (2014). Prevalence of anxiety disorders among Finnish primary care high utilizers and validation of Finnish translation of GAD-7 and GAD-2 screening tools. Scandinavian journal of primary health care, 32(2), 78-83.

Lemmens, J. S., Valkenburg, P. M., \& Peter, J. (2009). Development and validation of a game addiction scale for adolescents. Media psychology, 12(1), 77-95.

Männikkö, N., Billieux, J., \& Kääriäinen, M. (2015). Problematic digital gaming behavior and its relation to the psychological, social and physical health of Finnish adolescents and young adults. Journal of behavioral addictions, 4(4), 281-288.

Männikkö, N., Ruotsalainen, H., Tolvanen, A., \& Kääriäinen, M. (2019). Psychometric properties of the Internet Gaming Disorder Test (IGDT-10) and problematic gaming behavior 
among Finnish vocational school students. Scandinavian journal of psychology, 60(3), 252260.

Pontes, H. M., Schivinski, B., Sindermann, C., Li, M., Becker, B., Zhou, M., \& Montag, C. (2019). Measurement and conceptualization of gaming disorder according to the World Health Organization framework: The development of the Gaming Disorder Test.

International Journal of Mental Health and Addiction. doi:10.1007/s11469-019-00088-z Salonen, A. \& Raisamo, S. (2015) Suomalaisten rahapelaaminen 2015: Rahapelaaminen, rahapeliongelmat ja rahapelaamiseen liittyvät asenteet ja mielipiteet 15-74-vuotiailla. Helsinki: THL.

Spitzer, R. L., Kroenke, K., Williams, J. B., \& Löwe, B. (2006). A brief measure for assessing generalized anxiety disorder: the GAD-7. Archives of internal medicine, 166(10), 1092-1097. THL (2021) PROMIS: Yleinen terveys. Finnish Institute for Health and Welfare. https://thl.fi/fi/web/toimintakyky/toimintakyvyn-arviointi/kansallinen-promis-keskus von Bonsdorff, M. B., Seitsamo, J., Ilmarinen, J., von Bonsdorff, M. E., \& Taina, R. (2012). Work ability as a determinant of old age dis- ability severity: Evidence from the 28-year Finnish longitudinal study on municipal employees. Aging Clinical and Experimental Research, 24, 354-360. doi:10.1093/occmed/kqh005 Weiss, R.S. Loneliness. The experience of emotional and social isolation. Cambridge, MA:MIT Press 1973. 


\title{
Appendix 3 \\ Evolution of themes
}

\author{
Version 1 (after $1+1$ analyses)
}

\section{Treatment-seeker:}

i) Escaped discrimination and harrasment in adolescence to game worlds, which offered exploration, feelings to belong, and formed a strong identity around gaming

ii) Reflects on self-behaviour via ADHD and autistism in relatives; finds gaming to satisfy the constant need to do; boredom remains the most terrible feeling in existence

iii) A characteristic of a play experience is "hyperfixation", i.e. total engagement with the game world for 2-4 months (until it is exhausted); regulates gaming by choosing titles that "run out"

iv) Does not experience hyperfixation problematic as such, but in problematic life situations gaming becomes a coping mechanism cycle that makes one feel even worse

v) Identifies as introvert and gaming is a comfortable means to connect with others

\section{Esports player:}

i) Gaming formed his identity early on but never overtook other interests

ii) Needs for competition and learning are/were satisfied in gaming, which makes it meaningful (as "fun")

iii) Gaming is only a single (important) component in a rich life; life is satisfactory in many ways outside play too

iv) Awareness of gaming having the potential to overtake life, which makes one successfully balance and regulate play time

Version 2 (after $3+3$ analyses)

\section{Treatment-seekers:}

i) From early on, gaming has served as an escape from or solution to challenges in adolescence

ii) Over the years, constant difficulties to balance and regulate gaming with everyday life practices

iii) Attempts to seek pleasure from other sources, but gaming technologies satisfy various needs efficiently

Further themes strongly present for two participants, but not for the third:

a) The value of gaming perished gradually, resulting in life with no clear meaning

b) Just "gaming," rather than gaming being an identity

Further themes strongly present for the third participant, but not for the other two:

a) A characteristic of a play experience is "hyperfixation", i.e. total engagement until it exhaustion

b) In problematic life situations gaming becomes a coping mechanism cycle that makes one feel even worse

\section{Esports players:}

i) Gaming is only a single (important) part of a rich life; life is meaningful in many ways outside play too

ii) Gaming is a core component of identity and values

iii) Gaming has a paradoxical function as "fun" and "volunteer work", the latter of which manifests as rigid deliberate practice

iv) Awareness of gaming having the potential to overtake life, which has produced successful balancing and regulation of play 


\section{Version 3 (after all analyses)}

\section{Treatment-seekers:}

i) Escaping and solving difficulties in adolescence by gaming

ii) Living through the paradox of gaming satisfying many psychological needs but simultaneously having difficulties trying to reduce it

iii) Feeling of guilt or shame for having wasted time on gaming over life goals that would have had "real" value needs but simultaneously having difficulties trying to reduce it

iv) The experience of play, and the reflection as being a player is shadowed by past traumatic memories

\section{Esports players:}

i) Gaming is a core component of identity and values

ii) Awareness of gaming having the potential to overtake life, which has produced successful balancing and regulation of play

iii) Gaming is a high-investment meaningful routine

\section{Version 4 (meta-table) \\ For final edits negotiated with RK: https://osf.io/prwah/}

Meta-synthesis - treatment-seekers:

- Escaping and solving difficulties in adolescence by gaming; current play experience and their associations with self-image shadowed by memories

- Struggling with limited means to regulate gaming in a paradoxical situation where it both satisfies basic needs and contradicts personally meaningful obligations

- Feeling of guilt or regret for having wasted time on gaming over experiences that would have had "real value"

Meta-synthesis - esports players:

- Gaming is a core component of identity and values

- Awareness of gaming having the potential to become excessive, which has produced successful balancing and regulation of play

- Gaming is a high-investment meaningful routine

Meta-synthesis - group difference:

For treatment-seekers

- The experience of disorder derives from gaming interfering with self-what one wants to be, do, and have throughout life.

For esports players

- The experience of balance derives from gaming being integrated into self-what one wants to be, do, and have throughout life.

\section{All treatment-seekers:}

Aaron

- Simultaneously, play is valueless but also (the only) way to satisfy psychosocial needs; gaming has kept him away from satisfying them in other ways

- Intense play periods are responses to adverse events; a pattern since childhood bullying and depression

- Plays to regulate life and be in control, but experiences loss of control also in games

- Is gaming, rather than gaming being part of his identity 
- Since youth, has felt lonely and that he doesn't fit in, which led him to seek relatedness from gaming.

- Gaming became a core part of his identity and followed him, adjusting to different life challenges and keeping him connected with friends.

- Feels that sometimes gaming exceeds his emotional, mental, and physical resources, and leads to neglecting close social and other responsibilities.

- Thinks that non-online activities have higher value than gaming, and gaming leaves little time for them.

- Enjoys gaming but perceives it as an obstacle to proceed toward stable family life: finds it difficult to accept that "good old times are over."

Caius

- In single parent family, gaming time was not controlled and psychosocial needs were satisfied almost solely via digital play

- The meaningful joy of play gradually perished along with the diminishing competitive returns and turned into anger, frustration, and mindless repetition

- Does not identify as a gamer, but rather felt like a "relic" stuck in a MMO world while real life passed by

- Currently does not play but is negligent and disgusted by the traumatic emotions (end of romantic relationship) related to gaming

Dan

- Escaped discrimination and harassment in adolescence to game worlds, which offered exploration, feelings to belong, and formed a strong identity around gaming

- Reflects on self-behaviour via ADHD and autism in relatives; finds gaming to satisfy the constant need to $d o$; boredom remains the most terrible feeling in existence

- A characteristic of a play experience is "hyperfixation", i.e. total engagement with the game world for 2-4 months (until it is exhausted); regulates gaming by choosing titles that "run out"

- Does not experience hyperfixation problematic as such, but in problematic life situations gaming becomes a coping mechanism cycle that makes one feel even worse

- Identifies as introvert and gaming is a comfortable means to connect with others

Eetu

- In youth, was lonely and bullied, from which he escaped to excessively play games at home.

- Unlike in "real life", in single player games he had autonomy, competence, and control; in multiplayer games these elements were limited.

- Gaming became a primary means of life, but gradual perception of it as "useless" led him to try quitting.

- Work could give him gaming-like feelings when in a managerial position, and he dreams of finding a job that could provide such satisfaction.

- Feels that his addiction to play is permanent and is constantly afraid of relapse; thus rigidly controls access to all technologies.

- Gaming became an inseparable element of identity to the extent that gaming felt more real than other life

Fredrika

- In childhood and youth, was lonely and bullied at school. According to her retrospective reflection, she thus started playing games excessively.

- To seek acceptance (or avoid rejection), she continuously and obsessively pursued perfection in the meritocracy of the team game, which led to constant anxiety and stress.

- She felt lost outside of the game; not knowing what to do and what to pursue when not playing, and is now searching for herself.

- Feels that gaming is predictable and life is not; therefore gaming is safe and comfortable place.

- She feels that you cannot play too much, but rather you can do other things too little. The problem is when the only meaningful thing in your life is gaming. 


\section{All esports players:}

Gabriel

- Gaming formed his identity early on but never overtook other interests

- Needs for competition and learning are/were satisfied in gaming, which makes it meaningful (as "fun")

- Gaming is only a single (important) component in a rich life; life is satisfactory in many ways outside play too

- Awareness of gaming having the potential to overtake life, which makes one successfully balance and regulate play time

Heikki

- Strictly regulates gaming both emotionally and time-wise.

- Gaming has evolved from achievement-oriented into more social and fun, "doing it together" without people's background's interfering.

- Goes "all in" to everything and "overdoes" things, also games, which leads to "love-hate" relationships.

- Gaming allows him to discharge or burn energy; he needs an activity that stimulates him.

Ida

- Gaming has recently come to fill her long dream of competitive team sports

- Otherwise balanced life does not offer extreme challenge, which she seeks from gaming

- Play is heavily goal-oriented and structured to serve self-development as a player

- Experiences of gaming are unique because in them self-development happens with other people

Jaakko

- Single player gaming was among many adolescence hobbies, but eventually turned into more competitive and goal-oriented online play.

- Has always had a wide interest toward games and engages with all gaming media actively.

- Love-hate relationship with gaming, as he truly enjoys the competitive satisfaction of play, but hates the obsession that comes along.

- Over the years, has learned to enjoy and regulate his gaming so that it's now relaxed and does not lead to obsession.

Kalle

- Feels that he cannot play too much and never feels guilt or shame over gaming; gamin is his identity.

- Supported by teachers and parents, his gaming had an almost religious experience with MTG as a child, which made him feel finding a "home" and himself.

- He felt connected with other stigmatized players, which formed an identity; current gaming pleasures come partly from working in a team.

- He built his life around gaming, working for a company and spending almost all his time immersed in various gaming activities.

- Sports is a means of exercise, but you cannot do it as much due to injuries; the experience of gaming is accessible, fast, and easy.

Lauri

- Gaming is a goal-oriented part of daily routines and allows him to commit and invest.

- Plays all the available time (self-estimates up to $60,000 \mathrm{~h}$ of CS) but is balanced by other life interests and duties; does not struggle with self-regulation.

- Feels that gaming satisfies his competitive and social needs (self-identifies highly social) and enjoys specifically the psychology of teamwork.

- Feels now that in youth was playing too much and to an extent that it was "sick".

Mikko

- Throughout an economically unstable and mobile life, gaming has been an adaptable hobby that can follow him (also socially)

- Gaming has a paradoxical function as "fun" and "volunteer work", the latter of which manifests as rigid deliberate practice daily 
- The enjoyment of practice satisfies a constant need for competition and success, which work does not provide

- Gaming is a core component of identity and values, to an extent that exceeds even social relationships that clash with it

Niina

- Strong interest in technology and gaming since childhood, which did not fit the time's gender expectations.

- Built a strong gamer identity with a group of close male friends, which was not always easy but felt "right".

- Self-regulates play by trying to avoid excessively competitive gaming, and stops whenever the activity feels no more fun.

- She doesn't play because of the games but because of the empowering social interactions, which she couldn't have otherwise.

Oona

- Enjoys the coordination and organization in a team, in work and gaming.

- Does not enjoy competition, but competitive games provide the cooperative opportunities more than other types of play.

- "I don't have time to play too much" - lives a busy life with many ongoing projects, but still finds time to play $5 \mathrm{~h} /$ day.

- Has tried all possible hobbies, but gaming has remained throughout life because it's flexible and versatile.

- She "lives to learn", and gaming provides her the environment with infinite possibilities to learn new things.

Pasi

- Identifies as shy and gaming was thus a natural means to spend time, eventually leading to rich social networks as well.

- Experiences a strong need for challenge and competitiveness, yet does not satisfy the need by other means but gaming after sports injuries forced him to quit athletics.

- Has a solid gamer identity, which shows by the enjoyment of diverse gaming related products and experiences.

- Feels that gaming is in full control, and when he needs to reduce, he just does.

- Feels that gaming is a "holistic" activity for him, satisfying many needs at once, also as a means for reset stress. 